\title{
Room temperature commensurate charge density wave in epitaxial strained
}

\section{TiTe $_{2}$ multilayer films}

Sotirios Fragkos ${ }^{1}$, Roberto Sant ${ }^{2,3}$, Carlos Alvarez ${ }^{2,4}$, Alexei Bosak ${ }^{5}$, Polychronis Tsipas ${ }^{1}$, Dimitra Tsoutsou ${ }^{1}$, Hanako Okuno ${ }^{2,4}$, Gilles Renaud $^{2,4}$, Athanasios Dimoulas $^{1, *}$

${ }^{1}$ National Center for Scientific Research "Demokritos", 15310, Athens, Greece

${ }^{2}$ University Grenoble Alpes, Grenoble, France

${ }^{3}$ Néel Institute, CNRS, Grenoble, France

${ }^{4}$ INAC-MEM, CEA, Grenoble, France

${ }^{5}$ ESRF, The European Synchrotron Research Facility, Grenoble, France

\begin{abstract}
The group IVB 2D transition metal dichalcogenides (TMD) are considered to be stable in the high symmetry trigonal octahedral (1T) structure due to the lack of unpaired $d$ electrons on the metal site. It is found here that multilayer epitaxial TiTe 2 is an exception adopting a commensurate $2 \times 2 \times 2$ charge density wave (CDW) structure at room temperature with an ABA type of stacking as evidenced by direct lattice imaging and reciprocal space mapping. The CDW is stabilized by highly anisotropic strain imposed by the substrate with an out-off-plane compression which reduces the van der Waals gap increasing the interlayer coupling. A weaker $2 \times 2 \mathrm{CDW}$ is also confirmed at RT for epitaxial monolayer TiTe2. The addition of epitaxial strained TiTez to the family of CDW materials is expected to deepen our understanding on the CDW formation mechanisms in other TMD materials, still under debate and enable real world applications that take advantage of a CDW ground state at room temperature.
\end{abstract}


The group IVB 2D TMDs ${ }^{1,2,3}$ with the chemical formula $\mathrm{MX}_{2}(\mathrm{M}=\mathrm{Ti}, \mathrm{Hf}, \mathrm{Zr} ; \mathrm{X}=\mathrm{S}, \mathrm{Se}$, Te) are generally stable adopting a high symmetry trigonal octahedral (1T) structure ${ }^{4,5}$ since they do not possess unpaired $d$ electrons $^{6}$ at the metal site that could otherwise be a source of instability. A notable exception is $1 \mathrm{~T}-\mathrm{TiSe}_{2}$ which, below $205 \mathrm{~K}$ makes the transition to a lower symmetry commensurate CDW ground state associated with a $2 \times 2 \times 2$ periodic lattice distortion (PLD). Despite a large number of studies ${ }^{2,3}$ over the years since the first discovery ${ }^{7}$ and a couple of comprehensive reviews ${ }^{8,9}$ the actual mechanism for PLD/CDW formation is still under debate. The most recent experimental ${ }^{10-12}$ and theoretical ${ }^{13}$ works focus on the thickness dependence of CDW and the possible unconventional behavior in the ultimate 2D limit of a single layer $\mathrm{TiSe}_{2}$. On the other hand, the other $\mathrm{Ti}$ dichalcogenides namely $\mathrm{TiS}_{2}$ and $\mathrm{TiTe}_{2}$ did not show any clear evidence until very recently when a CDW state was reported only for $1 \mathrm{ML}$-thin $\mathrm{TiTe}_{2}$ at temperatures lower than $92 \mathrm{~K}^{14}$. It is surprising that the CDW in $\mathrm{TiTe}_{2}$ was found to be totally suppressed for films thicker than $1 \mathrm{ML}^{14}$, unlike the case of other TMDs where $1 \mathrm{ML}$ and bulklike films both make the transition to a CDW at nearly the same temperature.

The interest about $\mathrm{TiTe}_{2}$ is continuously increasing in view of theoretical predictions ${ }^{15}$ and more recent experimental evidence ${ }^{16}$ about pressure induced topological phase transitions in $\mathrm{TiTe}_{2}$. The possibility to also manipulate superconductivity by external pressure as predicted ${ }^{17}$ and more recently evidenced ${ }^{18}$ in bulk $\mathrm{TiTe}_{2}$ creates the prospect to explore the emergence of topological superconductivity in this material. In the latter work ${ }^{18}$ it has been shown that under non-hydrostatic pressure, a CDW-like state with estimated transition temperature above room temperature (RT) appears in bulk $\mathrm{TiTe}_{2}$ at around 0.5-1.8 GPa. These results call for a reexamination of the possibility to obtain a CDW in multilayer $\mathrm{TiTe}_{2}$ and indeed at RT with good potential for real world applications utilizing the properties of the CDW state ${ }^{19-22}$. 
In this paper it is shown that multilayer films $(50 \mathrm{ML} \sim 32 \mathrm{~nm})$, as well as single layer $\mathrm{TiTe}_{2}$ epitaxially grown on $\operatorname{InAs}(111) / \mathrm{Si}(111)$ substrates by molecular beam epitaxy exhibit, in ambient pressure conditions, a CDW distortion at room temperature which is sustained up to higher temperatures, at least $400{ }^{\circ} \mathrm{C}$ as evidenced by reflection high energy electron diffraction (RHEED) (Fig. S1). The results are explained in terms of anisotropic strain imposed by the substrate.

\section{Stability of 1T TiTe2: First principles calculations}

1T-TiTe 2 belongs to $\mathrm{P} \overline{3} \mathrm{~m} 1$ space group with trigonal symmetry assuming an octrahedral structure. The stability of $1 \mathrm{ML}$ and bulk $1 \mathrm{~T} \mathrm{TiTe} 2$ structure is studied by DFT and results on phonon dispersion are presented in Fig. 1a and S2. Employing Perdew-Burke-Ernzerhof (PBE) functionals, the $1 \mathrm{ML} 1 \mathrm{~T}$ structure is predicted to remain stable since only a small phonon softening at $\mathrm{M}$ occurs (Fig. S2) similar to previous report ${ }^{14}$. However, if Heyd-ScuseriaErnzerhof (HSE) hybrid functionals are used (see Methods) a large Kohn anomaly is predicted in one of the acoustic branches near M (Fig. 1a), implying a large 1T structural instability which could induce a transition to a lattice with lower symmetry. This is further supported by DFT/HSE energy minimization calculations in a $2 \times 2$ supercell predicting that the structure relaxes to a periodically distorted lattice as shown in Fig. $1 \mathrm{~b}$ which is more stable than the $1 \mathrm{~T}$ structure by 86 $\mathrm{meV}$ per unit cell. In the distorted lattice, one out of four Ti atoms in the $2 \times 2$ unit cell ( $\mathrm{Ti}_{\mathrm{I}}$ in Fig. 1b) remains fixed at the same position as in undistorted $1 \mathrm{~T}$ structure forming six equal bonds $(2.767 \AA)$ with the top and bottom Te atoms. On the other hand, the other three $\mathrm{Ti}$ atoms ( $\mathrm{Ti}$ III , in Fig. 1b) in the $2 \times 2$ cell are displaced from the ideal $1 \mathrm{~T}$ lattice positions by $0.021 a$ ( $a$ is the inplane lattice constant); Te atoms are also slightly displaced by $0.002 a$. Due to the TiII atom 
displacements, two short and two long bonds with lengths $2.701 \AA$ and $2.840 \AA$, respectively, are formed resulting in the configuration of Fig. $1 \mathrm{~b}$ which is similar to the $1 \mathrm{ML} \mathrm{TiSe}_{2} 2 \times 2$ commensurate CDW structure as previously reported ${ }^{7,13}$. In accordance with phonon calculations, when PBE functionals are used, energy minimization results in the $1 \mathrm{~T} \mathrm{TiTe}_{2}$ as the most stable structure. The structure is predicted to be essentially planar (confirmed by electron microscopy, Fig. 3 below) with no significant buckling and with all atoms lying on the lattice planes as in the undistorted $1 \mathrm{~T} \mathrm{TiTe}_{2}$.

The distorted lattice calculated by HSE hybrid functionals is found to be more stable by $90 \mathrm{meV}$ per unit cell than the $1 \mathrm{~T}$, also in the case where enlarged lattice parameters are considered as a result of tensile strain from the substrate as evidenced by GIXD measurements (see in the text below). The theoretical atomic fractional coordinates for the relaxed and strained $1 \mathrm{ML} \mathrm{TiTe}_{2}$ are given in tables $\mathrm{S} 1$ and $\mathrm{S} 2$, respectively.

\section{CDW order at the surface probed by in-situ scanning tunneling microscopy (STM)}

The surface structure probed by atomic resolution STM at RT and their corresponding fast Fourier transform (FFT) patterns are presented in Fig. 2. The 1 ML TiTe 2 STM image and $2 \times 2$ FFT (Fig. 2a, b) are very similar to data previously reported for relaxed $1 \mathrm{ML} \mathrm{TiTe}_{2}$ at $4.2 \mathrm{~K}$ and attributed to CDW superstructure ${ }^{14}$. The main difference is that in the present work the Moiré pattern attributed to the interaction with the InAs substrate is also visible. As expected, for the thicker $50 \mathrm{ML}$ film, the Moiré pattern is absent (Fig. 2c, d) since the top layer is not in direct contact with the substrate. Bright intensity modulation in a hexagonal formation (white arrows) in the real image indicates the doubling of periodicity (Fig. 2c) which however cannot be attributed to height differences between top Te atoms given that the Te plane is essentially flat 
as already discussed above and evidenced by STEM (see Fig. 3 below). Instead, this STM intensity modulation could be explained as charge density modulation associated with a transition to the low symmetry structure as depicted in Fig. 1b. It should be noted that the $2 \times 2$ superstructure signal in the FFT of the 50 ML (Fig. 2d) is significantly stronger compared to 1 ML film (Fig. 2b) which is relatively weak and diffuse, in agreement with the weak (and barely visible) superstructure streaks in RHEED (Fig S1). This indicates a much stronger CDW formation in the multilayer $\mathrm{TiTe}_{2}$ compared to $1 \mathrm{ML}$.

\section{Direct observation of CDW structure in multilayer TiTe2 by high resolution scanning tunneling spectroscopy (HR-STEM).}

STEM and nanobeam precession electron diffraction (N-PED) measurements are performed on the 50 ML-thick film and the main results are presented in Fig. 3 and in Fig. S3. The high-

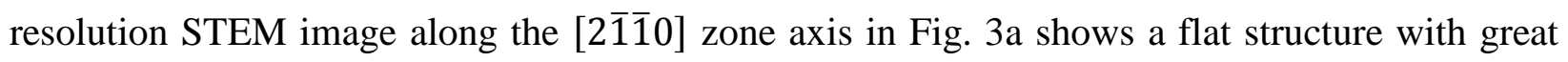
similarity to $1 \mathrm{~T} \mathrm{TiTe}_{2}$. However, a closer look reveals that the intensity of the Ti columns in Fig. 3a,c,d is modulated between sharp/intense (green arrows) and weak/diffuse (red arrows) essentially doubling the periodicity parallel to the layer. The same pattern appears along the vertical direction ( $c$-axis) where Ti column brightness is modulated between high and low values in two consecutive $\mathrm{TiTe}_{2}$ layers, thus doubling the periodicity along $c$ axis too, resulting in an overall $2 \times 2 \times 2$ periodicity in the film. This periodicity is also evident in the FFT (Fig. 3b) and the N-PED pattern (Fig. 3e) since weak superstructure spots, indicated by vertical blue thick arrows appear in between the main ones. The real image directly points to a CDW ABA type of stacking as shown in Fig. 3f where the aligned Ti atom columns A produce the sharp and bright Ti atom fringes (Fig. 3c,d) while the slightly misaligned atom columns B produce the weaker and diffuse 
Ti atom fringes (Fig. 3c,d). A simulation of the diffraction pattern using the CDW ABA structure of Fig 3f with fractional coordinates in tables S1 and S2 yields the configuration shown in Fig. 3g which matches very well the observed N-PED pattern (Fig. 3e) and the FFT (Fig. 3b) thus confirming that the $2 \times 2 \times 2$ periodic lattice distortion observed by STEM is the CDW-ABA structure depicted in Figs. $1 \mathrm{~b}$ and $3 \mathrm{f}$.

It is easily visible by a simple inspection of Fig. 3a that the Te-Ti-Te trilayers are separated by a very small $\mathrm{v} d$ Waals gap which is measured to be $\sim 3.1 \AA$ on average while

the Te-Ti-Te layer thickness is estimated to be $3.3 \AA$. The two quantities sum up to a lattice constant of the $1 \times 1 \operatorname{TiTe}_{2} c \sim 6.4 \AA$ which agrees well with the value of $c$ accurately measured by GIXD as discussed below.

\section{Reciprocal space mapping of CDW structure by Synchrotron grazing incidence x-ray diffraction (GIXD)}

Figure 4a,b show in-plane reciprocal space maps (RSM) for the $50 \mathrm{ML}$ InAs/TiTe 2 sample, for two values of the out-of-plane index $l=0.5$ (Fig. 4a) and $l=1$ (Fig. 4b), while $l=0$ and higher orders $(l=1.5,2,2.5)$ are shown in Fig. S4. The $l=0.5$ map shows the sharp superstructure spots which are attributed to the $\mathrm{CDW} \mathrm{TiTe}_{2}$ structure, located at the $\mathrm{L}$ points of the Brillouin zone (BZ) that have at least one in-plane index ( $h$ or $k$ ) half-integer. On the other hand the $l=1$ map mainly shows the very intense $1 \mathrm{~T} \mathrm{TiTe}_{2}$ Bragg peaks located at the $\Gamma$ points of the $\mathrm{BZ}$ with both $h$ and $k$ integer as well as much weaker CDW superstructure spots located at the M points. Considering also the data in Fig. S4, it can be said that, more generally, the half integer $l$ RSMs are dominated by the CDW superstructure peaks and the integer $l$ RSMs are dominated by the $1 \mathrm{~T}$ $\mathrm{TiTe}_{2}$ Bragg peaks. A diffuse elongated scattering pattern more visible in the half integer $l$ - 
planes (Fig.4 and S4) is attributed to thermal phonon diffuse scattering originating from Si substrate ${ }^{23}$.

The reciprocal space images of the $h 0 l$ and $h h l$ planes are shown in Figs $4 c$ and $4 d$, respectively. 1T $\mathrm{TiTe}_{2}$ Bragg peaks have integer $h l$ values, while $\mathrm{CDW}$ superstructure peaks have half-integer $h l$ values, clearly defining the $\times 2$ pattern along the in-and-out-of plane directions. The finite scattering intensity in the vertical direction (along $Г A$ ) which is more pronounced in the $h 0 l$ plane, is attributed to $\mathrm{Si}$ peak contributions (green arrows) and to finite sample thickness resulting in finite intensity in the half-integer $l$ RSMs (Fig.4a and S4b,d) at the $\Gamma$ points where it should normally be absent. The $h 0 l$ pattern is compatible with the FFT and NPED patterns observed in STEM (Fig. 3b,e).

Selected experimental $l$-scans are presented in Fig. 4e showing 1T $\mathrm{TiTe}_{2}$ (left panel) peaking at integer $l$ and CDW superstructure peaks (right panel) at half integer $l$. Order of magnitude lower intensity peaks at integer $l$ (shown by the vertical arrows in right panel of Fig. 4e), correspond to the M point weak superstructure spots as discussed above. In Fig. 4f the $l$ scans are simulated ${ }^{24,25}$ (for details see SI) using the CDW model with the ABA type of stacking (tables S1 and S2). A qualitative agreement with the experimental data of Fig. 4e can be seen, since the main features are reproduced at the correct $h k l$ positions within an order of magnitude agreement in intensity. The out of plane $\times 2$ periodicity manifested by the strongest superstructure peaks at half-integer $h k l$ is a direct consequence of the breaking of translation symmetry along $c$ axis in the ABA stacking configuration considered here. Markedly, AAA CDW stacking model fails to reproduce the experimentally observed $\times 2$ periodicity (data not shown).

The $2 \times 2 \times 2 \mathrm{CDW}$ reconstruction is a characteristic of our epitaxial $\mathrm{TiTe}_{2}$ films since bulk $\mathrm{TiTe}_{2}$ shows a stable $1 \mathrm{~T}$ structure where superstructure peaks at the L points of half integer $l$ 
RSMs are totally absent as can be seen from Fig. S5. Also by a direct comparison, it can be inferred that our epitaxial films exhibiting sharp diffraction spots are of superior quality compared to bulk (Fig. S5). Our films with low in-plane mosaic spread (see Fig. S6 and related discussion) are very well aligned with the substrate following the in-plane epitaxial relationship: InAs $[11 \overline{2}](111) / / \mathrm{TiTe}_{2}[10 \overline{1} 0](0001)$, despite the large lattice mismatch $(\sim-12 \%)$. The $1 \times 1$ $\mathrm{TiTe}_{2}$ in-plane $a$ and out-of- plane $c$ lattice parameters are accurately measured to be $a=3.842$ $( \pm 0.005) \AA$ in $1 \mathrm{ML} \mathrm{TiTe} 2$ and $a=3.862( \pm 0.003) \AA, c=6.349( \pm 0.008) \AA$ in $50 \mathrm{ML} \mathrm{TiTe} 2$. These values differ substantially from the experimentally determined free-standing bulk values $a=$ $3.777 \AA, c=6.498 \AA^{18,26}$. For the 50 ML film, the in-plane parameter $a$ is enlarged by $2.2 \%$ while the out of plane parameter $c$ is reduced by $2.3 \%$ indicative of epitaxial tensile strain in the film imposed by the substrate. Similarly, an epitaxial tensile strain of $1.7 \%$ can be inferred for the $1 \mathrm{ML} \mathrm{TiTe}_{2}$ film.

\section{Modifications in the electronic band structure imaged by in situ angle resolved photoemission spectroscopy (ARPES)}

The electronic band structure of $1 \mathrm{ML}$ (Fig. 5a) and $50 \mathrm{ML} \mathrm{TiTe}_{2}$ (Fig. 5b) are imaged by in-situ ARPES. They reveal a semimetalic material with two Te $5 p$ valence bands crossing $\mathrm{E}_{\mathrm{F}}$ near $\Gamma$, which overlap with Ti $3 \mathrm{~d}$ conduction bands at $\mathrm{M}(\mathrm{L}){ }^{27}$ by $0.2-0.3 \mathrm{eV}$, as expected from DFT calculations ${ }^{14}$ and experimental data ${ }^{27-29}$ of undistorted $1 \mathrm{~T} \mathrm{TiTe} 2$, suggesting that the PLD/CDW formation in our epitaxial films does not have a large influence on the electronic band structure. In contrast to common wisdom that a CDW should open a gap at the Fermi level $\left(\mathrm{E}_{\mathrm{f}}\right)$, no such gap is observed here. This is not in contradiction though with other CDW cases, as for example in $1 \mathrm{ML} \mathrm{TiTe} 2{ }_{2}{ }^{14}$ where valence and conduction bands cross at $\mathrm{E}_{\mathrm{f}}$, also in $\mathrm{TiSe}_{2}{ }^{10-12}$ where despite 
evidence for a small gap, $\mathrm{E}_{\mathrm{f}}$ often lies within one of the bands having finite density of states. It is worth noticing that the multilayer $\operatorname{TiTe}_{2}$ (Fig. 5b) with a stronger CDW structure compared to 1 ML as evidenced from Fig. 2, shows a lowering of one of the bands near $\Gamma$, about $0.25 \mathrm{eV}$ below $E_{f}$ (blue marks in Fig. 5b). This indicates a reduction of the density of states near $E_{f}$ corresponding to a partial gap opening (or a pseudogap) which could result in electronic energy gain, counterbalancing the losses associated with the PLD/CDW lattice distortions.

Notably, there is a weak and diffuse (blur) intensity near $\mathrm{E}_{\mathrm{f}}$ distributed along the $Г \mathrm{M}$ directions (E-k and contour plots in Figs.5 and S7), which could be considered as a dispersion-less band (DB), not predicted by theory. Similar dispersion-less intensity (blur) indicating localized conduction electrons has been observed previously in $\mathrm{TiTe}_{2}{ }^{28}$ and has been attributed to polaron effects associated with $\mathrm{CDW}$ order although it was not reproduced in later works $^{29}$.

It should be noted that overlapping of conduction and valence band was expected at $\mathrm{M}$ as a result of periodicity doubling and associated zone folding as previously reported $^{14}$ at $\mathrm{T}<92 \mathrm{~K}$ in synchrotron ARPES experiments. However such overlapping is not observed here possibly due to small spectral weights of the Te-p valence bands at M yielding signals beyond the sensitivity and resolution of our lab-based room temperature ARPES.

\section{Discussion and conclusions}

After several decades of research on CDW in 2D TMDs and the debate about the associated mechanisms, the common wisdom is that the softening of an acoustic phonon ${ }^{8,9,30}$ and the electron-phonon interaction are involved in the formation of a PLD and an associated CDW. In our multilayer $\mathrm{TiTe}_{2}$ the stronger intensity superstructure spots are obtained at the L points of the 
Brillouin zone (Fig. 4a) implying that softening of an acoustic phonon with wavevector $\vec{q}=\overrightarrow{\Gamma L}$ is at the heart of the commensurate $2 \times 2 \times 2 \mathrm{PLD} / \mathrm{CDW}$ formation in multilayer $\mathrm{TiTe}_{2}$ at $\mathrm{RT}$, similar to the $\mathrm{TiSe}_{2}$ case $^{7}$, while hints of localized conduction electrons at the Fermi surface from ARPES measurements (Fig.5) indicate polaronic effects ${ }^{27}$ likely associated with the CDW. Softening of an acoustic phonon with $\vec{q}=\overrightarrow{\Gamma M}$ as also predicted by theory (Fig. 1a) and evidenced by the very weak superstructure peaks at M (Fig. 4b, e) in GIXD could be responsible for the CDW observed at RT in $1 \mathrm{ML} \mathrm{TiTe}_{2}$ (Fig. 2a,b).

In free-standing $\mathrm{TiTe}_{2}$ multilayer films with relaxed lattice parameters ${ }^{14}$ and a sizeable v.d. Waals gap, the weakly coupled Te-Ti-Te (tri)layers do not favor the propagation of the CDW-driving $\vec{q}=\overrightarrow{\Gamma L}$ phonons through the layers, probably explaining the absence of CDW in multilayer films ${ }^{14}$. In our epitaxial films though the situation is different, because a significant interaction with the substrate is established producing an anisotropic strain such that the in-plane parameter is enlarged and the out of plane parameter along the $c$-axis is compressed by $2.3 \%$ as accurately measured by synchrotron GIXD, yielding an estimated stress $\sim 0.6-0.8 \mathrm{GPa}$ if a measured $^{31}$ bulk modulus $B \sim 0.28-0.40 \mathrm{GPa}$ is assumed. The compression results in an appreciable reduction of van der Waals gap from $~ 3.2 \AA^{18}$ to the value of $3.1 \AA$ that we measured by STEM bringing the Te-Ti-Te trilayers closer between each other thus enhancing interlayer Te-Te interactions, signifying a transition from a $2 \mathrm{D}$ to a $3 \mathrm{D}-\mathrm{like} \mathrm{TiTe}_{2}$ crystal. We anticipate that this transition to a 3D structure facilitates the propagation of the important phonon modes along $\Gamma \mathrm{L}$ which are required to propagate across tightly coupled $\mathrm{TiTe}_{2}$ layers in thick films or bulk material to provide the source of instability that drives the CDW distortion at RT which is sustained up to $400{ }^{\circ} \mathrm{C}$. 
The analysis based on the anisotropic strain correlates well with predictions ${ }^{32}$ in $\mathrm{TiSe}_{2}$ stating that the CDW critical temperature is enhanced under biaxial tensile (stretching) strain (compression along the c-axis). Our analysis correlates also nicely with the recent experimental results ${ }^{18}$ reporting the emergence of $\mathrm{CDW}$ in a $\mathrm{TiTe}_{2}$ multilayer under $1.8 \mathrm{GPa}$ non-hydrostatic pressure (effectively uniaxial compression along the $c$-axis as in our films) also providing hints of a re-entrant $2 \times 2 \mathrm{CDW}$ with an estimated critical temperature above RT. The latter appears during the decompression phase around $0.5 \mathrm{GPa}$, which is similar in magnitude to the epitaxial stress exerted on our films (0.6-0.8 GPa, see above).

\section{Methods:}

Growth The In-terminated-InAs(111)/Si(111) wafers prepared by MOCVD are used as the substrate materials. The substrate are chemically treated in a solution of $\mathrm{HCl}(5 \mathrm{~N})$ in isopropyl alcohol and subsequent dipping in isopropyl alcohol, followed by an annealing step at $400{ }^{\circ} \mathrm{C}$ in UHV until a $2 \times 2$ superstructure is obtained by RHEED, indicating a clean, oxide-free surface. The molecular beam epitaxy (MBE) of thin $\mathrm{TiTe}_{2}$ films was performed in an ultra-high vacuum (UHV) chamber at $400{ }^{\circ} \mathrm{C}$. High purity metal Ti was evaporated from an e-gun evaporator whereas Te was evaporated from effusion cells. $\mathrm{TiTe}_{2}$ epilayers were grown under Te rich conditions with a growth rate of $1 \mathrm{ML} / 30 \mathrm{sec}$.

Scanning Tunneling Microscopy The surface morphology of the $\mathrm{TiTe}_{2}$ epilayers on InAs(111) is examined by in-situ RT Omicron UHV-STM. The scanning conditions are $I_{t}=500 \mathrm{nA}, V_{b}$ $=+0.05 \mathrm{~V}$, loop gain $1.7 . V_{b}$ in our system is applied on the sample so that positive $V_{b}$ means empty state STM images. 
Electron Microscopy. STEM and N-PED experiments were performed using a probe-corrected FEI Titan Themis S/TEM operated at $200 \mathrm{keV}$. High angle annular dark field STEM images were collected using a convergence semi-angle of $18 \mathrm{mrad}$ with the annular detector collecting scattering above 65 mrad. N-PED was collected using the FEI Epsilon acquisition software, and more details about the method can be found by these works ${ }^{33,34}$. The N-PED data was acquired using a convergence semi-angle of $1.5 \mathrm{mrad}$, a precession angle of 0.3 degrees, and precession frequency of $200 \mathrm{~Hz}$. Electron diffraction patterns were simulated using the JEMS software package. The specimen was prepared by focused ion beam lift-out protocol using a FEI dualbeam Strata 400S.

Synchrotron GIXD Measurements: Diffraction measurements were performed at the European Synchrotron Radiation Facility by means of the UHV-MBE CVD diffractometer installed at the BM32 CRG/IF, optimized for GIXD, and ID28 diffraction side station. The experimental setup energy and incident angle were set at $11 \mathrm{keV}(1.13 \AA)$ and $0.2^{\circ}$, the latter set slightly below the critical angle value for total reflection in order to enhance the 2D film signal while minimizing the background. During the measurements the sample was maintained under UHV (base pressure: $3 \times 10^{-3} \mathrm{mbar}$ ) in the UHV chamber that is coupled to the diffractometer. ID28 data were collected with the fixed incidence angle $1 \mathrm{deg}$. at $17.8 \mathrm{keV}(0.697 \AA)$ on the sample rotating around its normal; scattering intensity was collected by PILATUS3X 1M detector in shutterless mode with angular step of $0.25 \mathrm{deg}$. CrysAlis package ${ }^{35}$ was employed for refinement of experiment geometry, high qualiry reciprocal space reconstructions were produced with locally developed software. 
Photoelectron spectroscopy. ARPES measurements were carried out with a PHOIBOS 100 (SPECS) hemispherical analyzer, made of $\mu$-metal at a pass energy of $15 \mathrm{eV}$, using a 2D CCD detector and a He plasma source with He I excitation energy at $21.22 \mathrm{eV}$. The energy resolution of the system was better than $40 \mathrm{meV}$, although the measurement resolution is limited by thermal excitation processes to around $100 \mathrm{meV}$.

First Principles Calculations. Density Functional Theory calculations were performed using the Vienna $\mathrm{Ab}$ initio Simulation Package $(\mathrm{VASP})^{36-38}$ and projector-augmented waves ${ }^{39,40}$. The Heyd-Scuseria-Ernzerhof (HSE06) hybrid functionals ${ }^{41}$ were used as the exchange correlation potential to determine the atomic displacements in the (2×2) CDW $\mathrm{TiTe}_{2}$ structure, total energy and phonon dispersion calculations. Atomic positions were optimized until the residual forces

were less than $0.005 \mathrm{eV} \cdot \AA^{-1}$. Also, a vacuum $15 \AA$ was used to avoid interaction between the periodically repeated layers. The plane wave kinetic energy cutoff was set at $500 \mathrm{eV}$, employing a $16 \times 16 \times 1(8 \times 8 \times 1)$ k-mesh for the normal $(\mathrm{CDW})$ phase. Perdew-Burke-Ernzerhof functional ${ }^{42}$ was also used for the phonon dispersion calculation of the $(1 \times 1) 1 \mathrm{~T}-\mathrm{TiTe} 2$ structure. Phonon calculations were performed using the Phonopy package ${ }^{43}$.

\section{References}

1. Manzeli, S., Ovchinnikov, D., Pasquier, D., Yazyev, O. V. \& Kis, A. 2D transition metal dichalcogenides. Nat. Rev. Mater. 2, 17033 (2017).

2. Han, G. H., Duong, D. L., Keum, D. H., Yun, S. J. \& Lee, Y. H. van der Waals Metallic Transition Metal Dichalcogenides. Chem. Rev. 118, 6297-6336 (2018). 
3. Yan, C., Gong, C,. Wangyang, P., Chu, J., Hu, K., Li, C., Wang, X., Du, X., Zhai, T., Li, Y. \& Xiong, J. 2D Group IVB Transition Metal Dichalcogenides. Adv. Funct. Mater. 1803305 (2018).

4. Giamini, S. A., Velasco, J. M., Tsipas, P., Tsoutsou, D., Renaud, G. \& Dimoulas, A. Molecular Beam Epitaxy of thin $\mathrm{HfTe}_{2}$ semimetal films. 2D Mater. 4, 4015001 (2017).

5. Tsipas, P., Tsoutsou, D., Fragkos, S., Sant, R., Alvarez, C., Okuno, H., Renaud, G., Alcotte, R., Baron, T. \& Dimoulas, A. Massless Dirac Fermions in $\mathrm{ZrTe}_{2}$ Semimetal Grown on InAs(111) by van der Waals Epitaxy. ACS Nano 12, 1696-1703 (2018).

6. Yang, H., Kim, S. W., Chhowalla, M. \& Lee, Y. H. Erratum: Structural and quantumstate phase transitions in van der Waals layered materials. Nat. Phys. 13, 931-937 (2017).

7. Di Salvo, F. J., Moncton, D. E. \& Waszczak, J. V., Electronic properties and superlattice formation in the semimetal TiSe2. Phys. Rev B 14, 4321 (1977).

8. Rossnagel, K. On the origin of charge-density waves in select layered transition-metal dichalcogenides. J. Phys. Cond. Mater. 23, 213001 (2011).

9. Zhu, X., Cao, Y., Zhang, J., Plummer, E. W. Z \& Guo, J. Classification of charge density waves based on their nature. PNAS, 112, 2367 (2015).

10. Chen, P., Chan, Y.-H., Fang, X.-Y., Zhang, Y., Chou, M. Y., Mo, S.-K, Hussain, Z., Fedorov, A.-V. \& Chiang, T.-C. Charge density wave transition in single-layer titanium diselenide. Nat. Commun. 6, 8943 (2015).

11. Chen, P., Chan, Y.-H., Wong, M.-H., Fang, X.-Y., Chou, M. Y., Mo, S.-K, Hussain, Z., Fedorov, A.-V. \& Chiang, T.-C. Dimensional Effects on the Charge Density Waves in Ultrathin Films of TiSe 2 . Nano Lett. 16, 6331-6336 (2016). 
12. Sugawara, K., Nakata, Y., Shimizu, R., Han, P., Hitosugi, T., Sato, T., \& Takahashi, T. Unconventional Charge-Density-Wave Transition in Monolayer 1T-TiSe 2 . ACS Nano 10, $1341-1345$ (2016).

13. Guster, B., Canadell, E., Pruneda, M. \& Ordejon, P. First principles analysis of the CDW instability of single-layer $1 \mathrm{~T}-\mathrm{TiSe}_{2}$ and its evolution with charge carrier density. $2 D$ Mater. 5, 025024 (2018).

14. Chen, P., Pai, W. W., Chan, Y.-H., Takayama, A., Xu, C.-Z., Karn, A., Hasegawa, S., Chou, M. Y., Mo, Z., Fedorov, A.-V. \& Chiang, T.-C. Emergence of charge density waves and a pseudogap in single-layer TiTe2. Nat. Commun. 8, 516 (2017).

15. Zhang, Q., Cheng, Y. \& Schwingenschlögl, U. Series of topological phase transitions in $\mathrm{TiTe}_{2}$ under strain. Phys. Rev. B 88, 155317 (2013).

16. Zhang, M., Wang, X., Rahman, A., Zeng, Q., Huang, D., Dai, R., Wang, Z., \& Zhang, Z. Pressure-induced topological phase transitions and structural transition in $1 \mathrm{~T}-\mathrm{TiTe}_{2}$ single crystal. Appl. Phys. Lett. 112, 041907 (2018).

17. Xiao, R. C., Lu, W. J., Shao, D. F., Li, J. Y., Wei, M. J., Lv, H. Y., Tong, P., Zhua X. B. \& Sun, Y. P. Manipulating superconductivity of 1T-TiTe 2 by high pressure. J. Mater. Chemistry C 5, 4167-4173 (2017).

18. Dutta, U., Malavi P.S., Sahoo S., Joseph, B. \& Karmakar, S. Pressure-induced superconductivity in semimetallic $1 \mathrm{~T}-\mathrm{TiTe}_{2}$ and its persistence upon decompression Phys. Rev. B 97, 060503 (2018).

19. Liu, G., Debnath, B., Pope, T. R., Salguero, T. T., Lake, R. K., Balandin, A. A. A chargedensity-wave oscillator based on an integrated tantalum disulfide-boron nitride-graphene device operating at room temperature. Nat. Nanotech. 11, 845-850 (2016). 
20. Vaskivskyi, I., Mihailovic, I. A., Brazovskii, S., Gospodaric, J., Mertelj, T., Svetin, D., Sutar, P., Mihailovic, D. Fast electronic resistance switching involving hidden charge density wave states. Nat. Commun. 7, 11442 (2016).

21. Hollander, M. J., Liu, Y., Lu, W.-J., Li, L.-J, Sun, Y.-P., Robinson, J. A., \& Datta, S. Electrically Driven Reversible Insulator-Metal Phase Transition in $1 \mathrm{~T}-\mathrm{TaS}_{2 .}$ Nano Lett. 15, 1861-1866 (2015).

22. Khan, J., Nolen, C. M., Teweldebrhan, D., Wickramaratne, D., Lake, R. K. \& Balandin, A. A. Anomalous electron transport in back-gated field-effect transistors with $\mathrm{TiTe}_{2}$ semimetal thin-film channels. Appl. Phys. Lett. 100, 043109 (2012).

23. Holt, M., Wu, Z., Hong, H., Zschack, P., Jemian, P., Tischler, J., Chen, H. \& Chiang, T.C. Determination of phonon dispersions from X-Ray transmission scattering: The example of Silicon. Phys. Rev. Lett. 83, 3317 (1999).

24. Drnec, J., Zhou, T., Pintea, S., Onderwaater, W., Vlieg, E., Renaud, G. \& Felici, R. Integration techniques for surface X-ray diffraction data obtained with a two-dimensional detector. J. Appl. Cryst. 47, 365-377 (2014).

25. Vlieg, E. ROD: a program for surface X-ray crystallography. J. Appl. Cryst. 33, 401-405 (2000).

26. Arnaud, Y. \& Chevreton, M. Etude comparative des composés TiX $(X=S, S e, T e)$. Structures de $\mathrm{TiTe}_{2}$ et TiSeTe. J. Sol. State Chem. 39, 230-239 (1981).

27. Rossnagel, K., Kipp, L., Skibowski, M., Solterbeck, C., Strasser, T., Schattke, W., Voß, D., Krüger, P., Mazur, A. \& Pollmann, J. Three-dimensional Fermi surface determination by angle-resolved photoelectron spectroscopy. Phys. Rev B 63, 125104 (2001). 
28. De Boer, D. K. G., van Bruggen, C. F., Bus, G. W., Coehoorn, R., Haas, C., Sawatzky, G. A., Myron, H. W., Norman, D. \& Padmore H., Titanium ditelluride: Band structure, photoemission, and electrical and magnetic properties, Phys. Rev B 29, 6797 (1984).

29. Claessen, R., Anderson, R. O., Gweon, G.-H., Allen, J.W., Ellis, W.P., Janowitz C., Olson, C. G., Shen, Z. X., Eyert, V., Skibowski, M., Friemelt, K., Bucher, E., and Hüfner, S. Complete band-structure determination of the quasi-two-dimensional Fermi-liquid reference compound TiTe2. Phys. Rev. B 54, 2453 (1996).

30. Yoshida, Y. \& Motizuki, K. Electron Lattice Interaction and Lattice Instability of 1TTiSe $_{2}$, J. Phys. Soc. Jpn. 49, 898-905 (1980).

31. Rajaji, V., Dutta, U., Sreeparvathy, P. C., Sarma, S. C., Sorb, Y. A., Joseph, B., Sahoo, S., Peter, S. C., Kanchana, V. \& Narayana, C., Structural, vibrational, and electrical properties of 1T-TiTe 2 under hydrostatic pressure: Experiments and theory. Phys. Rev. B 97, 085107 (2018).

32. Fu, Z.-G., Hu, Z.-Y., Yang, Y., Lu, Y., Zheng, F.-W. \& Zhang, P. Modulation of doping and biaxial strain on the transition temperature of the charge density wave transition in 1T-TiSe 2. RSC Adv. 6, 76972 (2016).

33. Béché, A., Rouvière, J. L., Clément, L. \& Hartmann, J. M. Improved precision in strain measurement using nanobeam electron diffraction. Appl. Phys. Lett. 95, 123114 (2009).

34. Cooper, D., Bernier, N. \& Rouvière, J.-L. Combining $2 \mathrm{~nm}$ spatial resolution and $0.02 \%$ precision for deformation mapping of semiconductor specimens in a transmission electron microscope by precession electron diffraction. Nano Lett. 15, 5289-5294 (2015).

35. Oxford Diffraction /Agilent Technologies UK Ltd, CrysAlisPRO, Yarnton, England 
36. Kresse, G. \& Hafner, J. Ab initio molecular dynamics for open-shell transition metals. Phys. Rev. B 48, 13115-13118 (1993).

37. Kresse, G. \& Furthmüller, J. Efficiency of ab-initio total energy calculations for metals and semiconductors using a plane-wave basis set. J. Comput. Mater. Sci. 6, 15-50 (1996).

38. Kresse, G. \& Furthmüller, J. Efficient iterative schemes for ab initio total-energy calculations using a plane-wave basis set. Phys. Rev. B 54, 11169-11186 (1996).

39. Blöchl, P. E. Projector augmented-wave method. Phys. Rev. B 50, 17953-17979 (1994).

40. Kresse, G. \& Joubert, J. From ultrasoft pseudopotentials to the projector augmented-wave method. Phys. Rev. B 59, 1758-1775 (1999).

41. Heyd, J., Scuseria, G. E. \& Ernzerhof, M. Hybrid functionals based on a screened Coulomb potential. J. Chem. Phys. 118, 8207-8215 (2003).

42. Perdew, J. P., Burke, K. \& Ernzerhof, M. Generalized gradient approximation made simple. Phys. Rev. Lett. 77, 3865-3868 (1996).

43. Togo, A. \& Tanaka, I. First principles phonon calculations in materials science. Scr. Mater. 108, 1-5 (2015).

\section{Acknowledgements}

This work was financially supported by the LANEF Chair of Excellence program of U. Grenoble Alpes and CEA (A.D.), the Greek State Scholarships Foundation (IKY) Program for the strengthening of postdoctoral research (P.T.), the project MIS 5002567, co-financed by Greece and the EU (European Regional Development Fund) (D.T), the French state fundsANR-10-LABX-51-01 (Labex LANEF du Programmed'Investissements d'Avenir) and Equipex ANR-11-EQPX-0010 (G.R.). The authors thank Dr. P. Rodiere of CNRS/Neel Institute for very useful discussions on 
soft phonon mediated X-Ray scattering and Dr. T. Baron of Université Grenoble Alpes, CNRS, CEA/Leti Minatec, LTM for providing us with the InAs/Si substrates.

\section{Author contributions}

Sotirios Fragkos performed DFT calculations and was involved in ARPES, Roberto Sant, Alexei

Bosak, and Gilles Renaud performed the GIXD, Carlos Alvarez and Hanako Okuno performed

STEM, P. Tsipas performed the MBE growth, RHEED and STM, D. Tsoutsou performed

ARPES and A. Dimoulas was involved in DFT, MBE growth, STM and ARPES

\section{Competing Interests}

The authors declare no competing interests.

\section{Materials \& Correspondence}

Should be addressed to Athanasios Dimoulas 


\section{Figures}

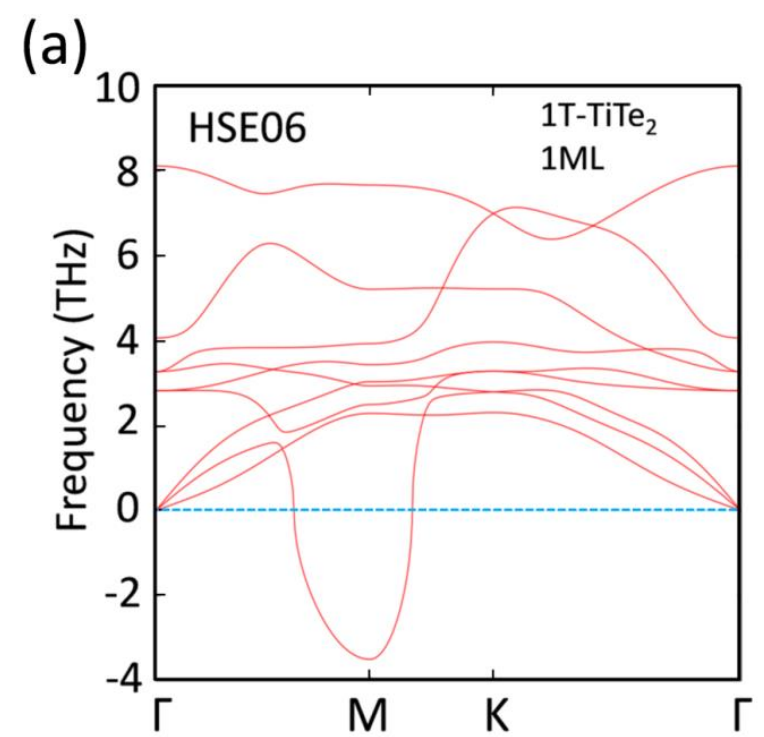

(b)

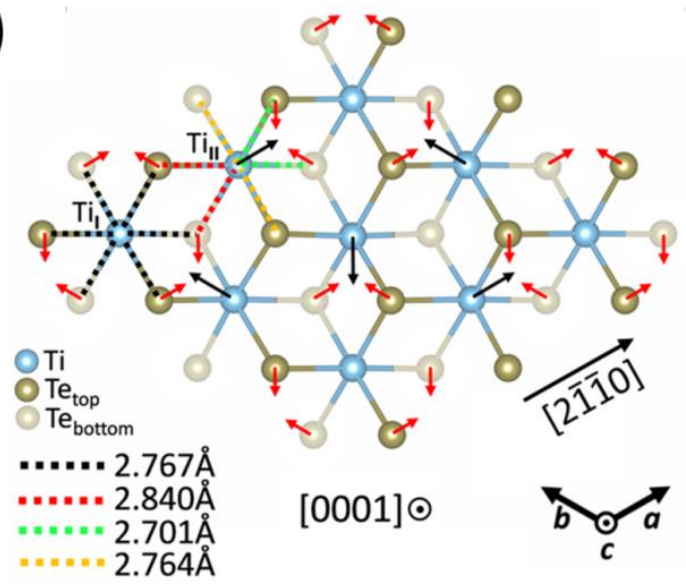

Fig. 1. Stability of the 1 ML 1T-TiTe2. (a) Phonon dispersion calculated by DFT using HSE hybrid functionals. The large and wide range of negative frequencies around $\mathrm{M}$, represent imaginary frequency values indicative of structural instability. (b) CDW $2 \times 2$ atom configuration in 1 ML $\mathrm{TiTe}_{2}$. $\mathrm{Ti}_{\mathrm{I}}$ and $\mathrm{Ti}_{\text {II }}$ show undisplaced and displaced Ti atoms, respectively. Long black and short red arrows show the direction of $\mathrm{Ti}$ and $\mathrm{Te}$ atom displacements, respectively. The dotted colored lines show Ti-Te bonds with different lengths. 

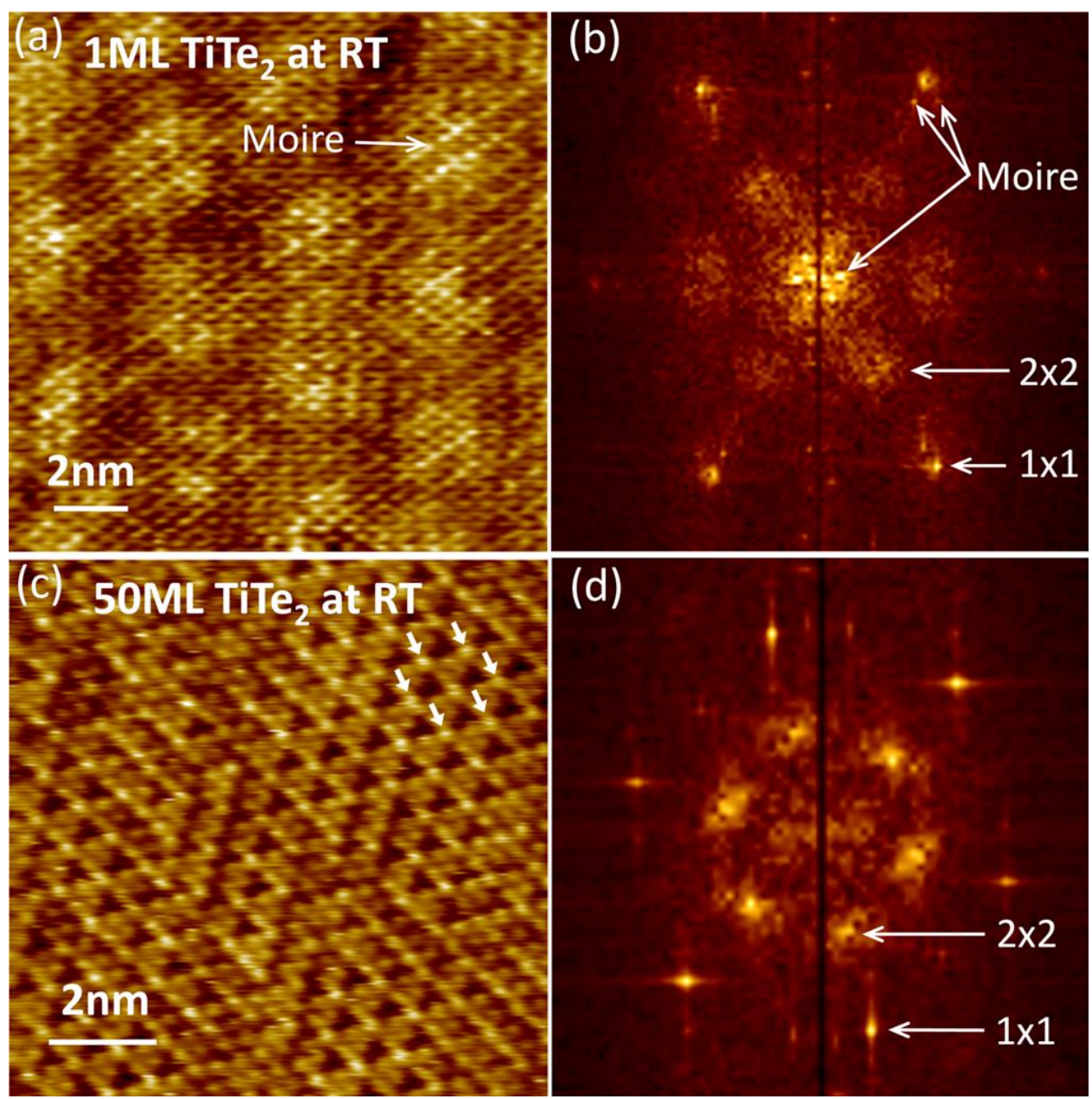

Fig. 2. Surface ordering of epitaxial TiTe 2 by in-situ STM at room temperature. Images are taken at constant current mode, $I_{t}=500 \mathrm{nA}, V_{b}=+0.05 \mathrm{~V}$, loop gain 1.7. $1 \mathrm{ML}^{\mathrm{T} i T e} \mathrm{STM}_{2} \mathrm{STM}$ (a) and FFT (b). In (b) the hexagonal formation of diffraction spots at the center is the Moiré pattern which is rotationally aligned with the $(1 \times 1) \mathrm{TiTe}_{2}$ lattice. Two of the Moiré peaks are also visible near the $(1 \times 1)$ diffraction spot. 50 ML $\mathrm{TiTe}_{2}$ STM (c) and corresponding FFT (d), showing bright and sharp $2 \times 2$ superstructure spots. The white arrows in (c) indicate atomic positions with enhanced intensity marking the $2 \times 2$ periodicity. 


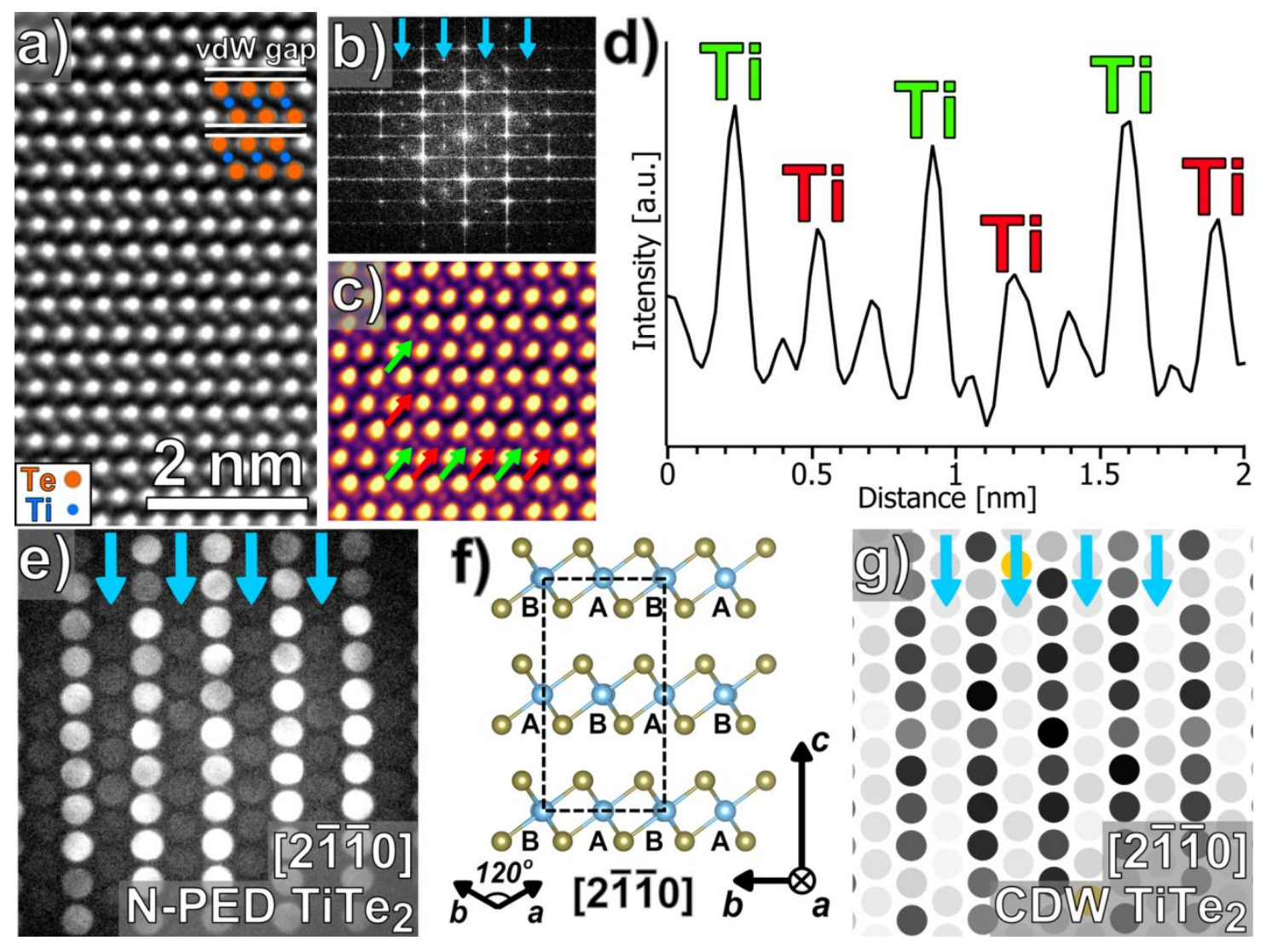

Fig. 3: HR STEM and diffraction pattern simulation. (a) STEM image of $\mathrm{TiTe}_{2}$ film along the [2 $\left.\overline{1} \overline{1} 0\right]$ zone axis. Bright large spots are Te and weak small spots are Ti atom columns. (b) FFT corresponding to (a). (c) False-color STEM image with arrows indicating Ti columns of different intensity, and (d) intensity line scan plot across row of $\mathrm{Ti}$ atomic columns showing the intensity modulation. (e) PED pattern of $\mathrm{TiTe}_{2}$ film along the [2 $\left.\overline{1} \overline{1} 0\right]$ zone axis. (f) Layer stacking in bulk CDW. A and B mark the Ti atomic columns which consist of atoms aligned (A) and slightly misaligned (B) along the [2 $\overline{1} \overline{1} 0]$ direction. The dashed line is the projection of the $2 \times 2$ unit cell along the [2$\overline{1} \overline{1} 0]$ direction, showing that two layers belong to one unit cell. The second layer is displaced with respect to the first (bottom) by 0.5 unit cell resulting in a $2 \times 2 \times 2$ periodicity. (g) Simulated diffraction pattern of $2 \times 2 \times 2 \mathrm{CDW} \mathrm{TiTe}_{2}$ according to the ABA stacking model in (f). The blue thick vertical arrows in (b), (e) and (g) indicate the additional diffraction spots associated with the periodicity doubling in the CDW structure caused by the shift of the Ti atoms. Double diffraction spots indicated in yellow in $(\mathrm{g})$ are rediffraction in a thick crystal or between two crystals. 

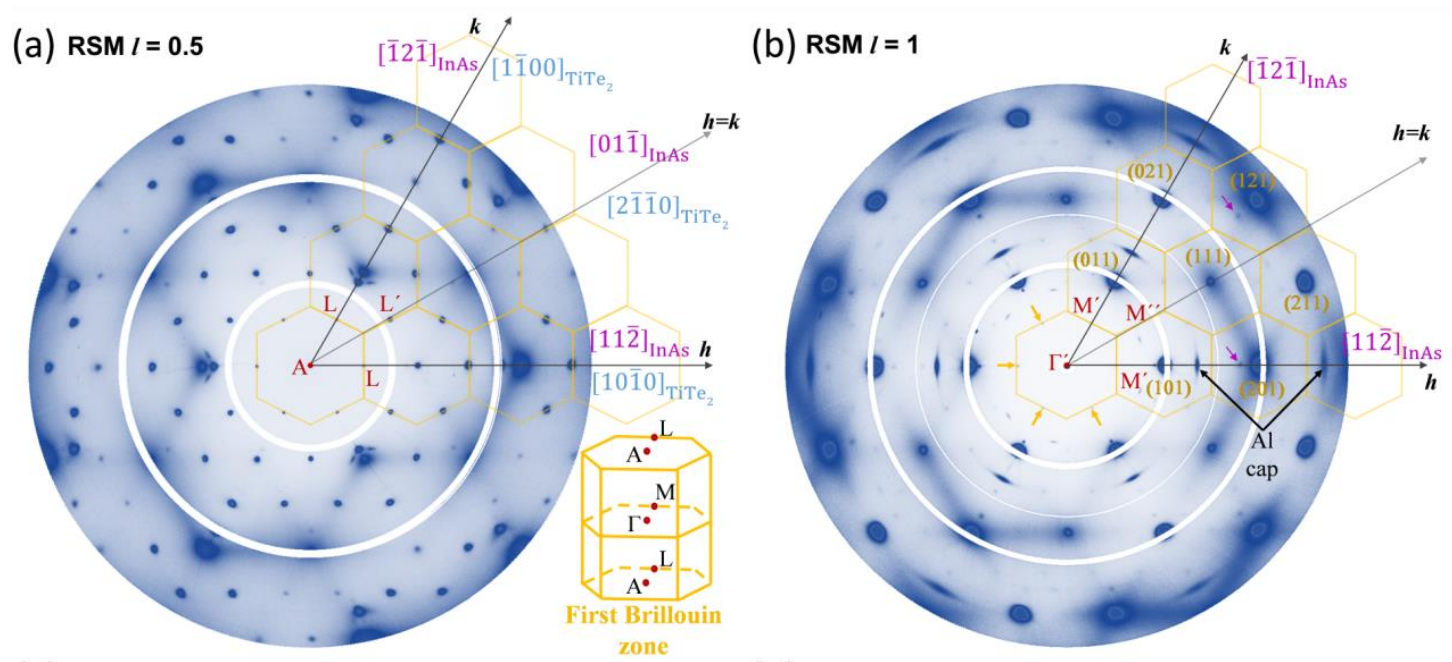

(c)

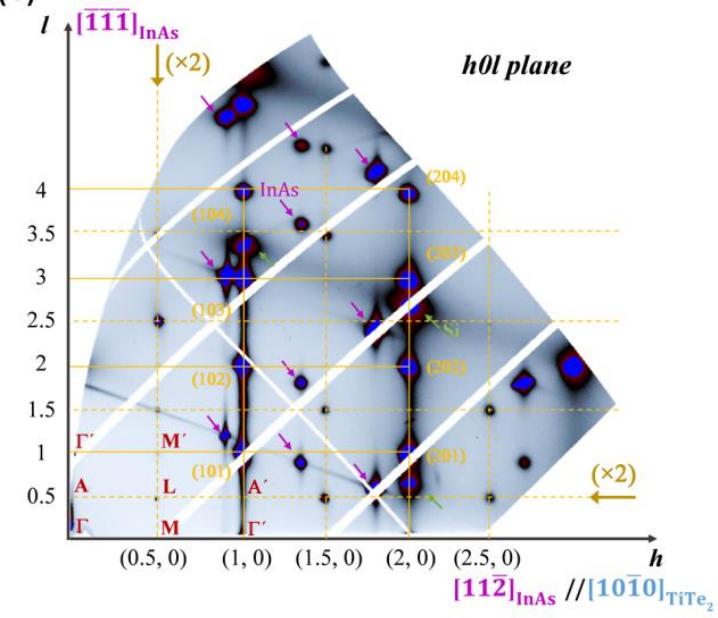

(e) Experimental $l$-scans

(d)
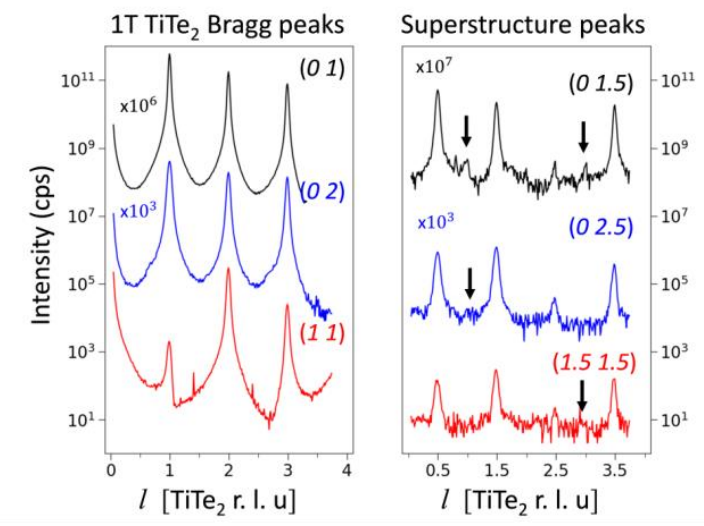

(f)

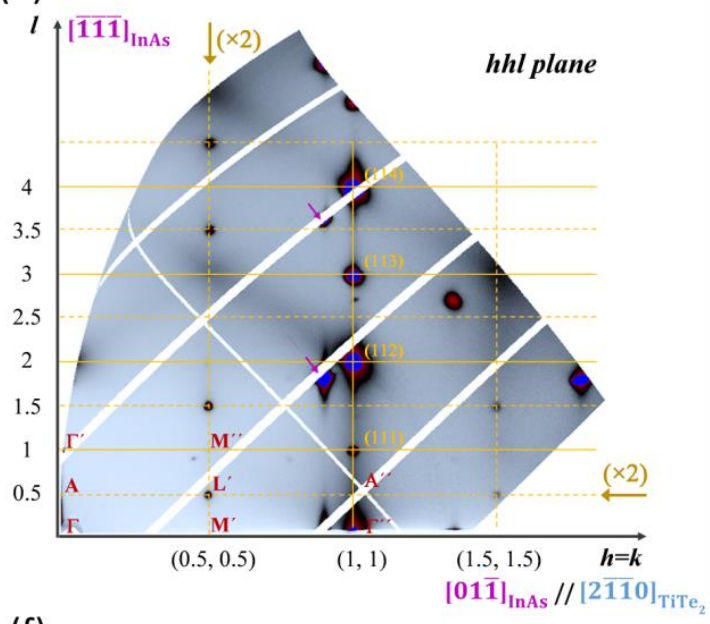
l-scans simulations

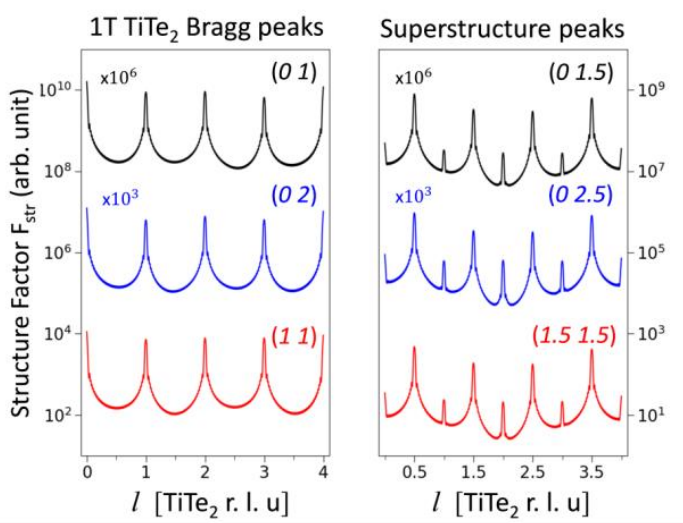

Fig. 4 Reciprocal space mapping of CDW 50 ML TiTe 2 . (a) RSM for $l=0.5$ (a) and $l=1$ (b). The honeycomb-like yellow grid shows the projection of the Brillouin zone (BZ) structure (inset) with red letters marking high symmetry points of the first BZ and neighboring BZ (primed and double primed). 
Yellow numbers in (b) show surface Miller indices of $1 \mathrm{~T} \mathrm{TiTe}_{2}$ Bragg peaks located at the center $(\Gamma$ points) of BZ, in contrast to superstructure spots (not indexed) in (a) which are located at the edge (L points) of the BZ. Yellow arrows in (b) show weak superstructure peaks located at the M points of the BZ while magenta arrows show InAs diffraction peaks. Imaging of portion of the $h 0 l$ (c) and $h h l$ planes (d). The $1 \mathrm{~T} \mathrm{TiTe}_{2}$ Bragg peaks are located at the crosspoints of the vertical and horizontal yellow solid lines (guides to the eye) and have all integer $h k l$ values. The superstructure (satellite) peaks are located at the crosspoints of the vertical and horizontal yellow dotted lines and have half integer $h k l$ values. The magenta and green arrows show Bragg peaks of InAs and Si, respectively. (e) The experimental $l$-scans for a selection of $(h k)$ pairs showing peaks at integer or half integer $l$ for $1 \mathrm{~T} \mathrm{TiTe}_{2}$ Bragg and superstructure peaks, respectively. The black vertical arrows in the right panel indicate very weak superstructure peaks at integer $l$ which are associated with the weak spots at M points seen integer $l$ RSMs. (f) Simulation of $l$-scans in terms of the structure factor using the CDW - ABA stacking model showing qualitative agreement with the experimental data in (e). 
(a)
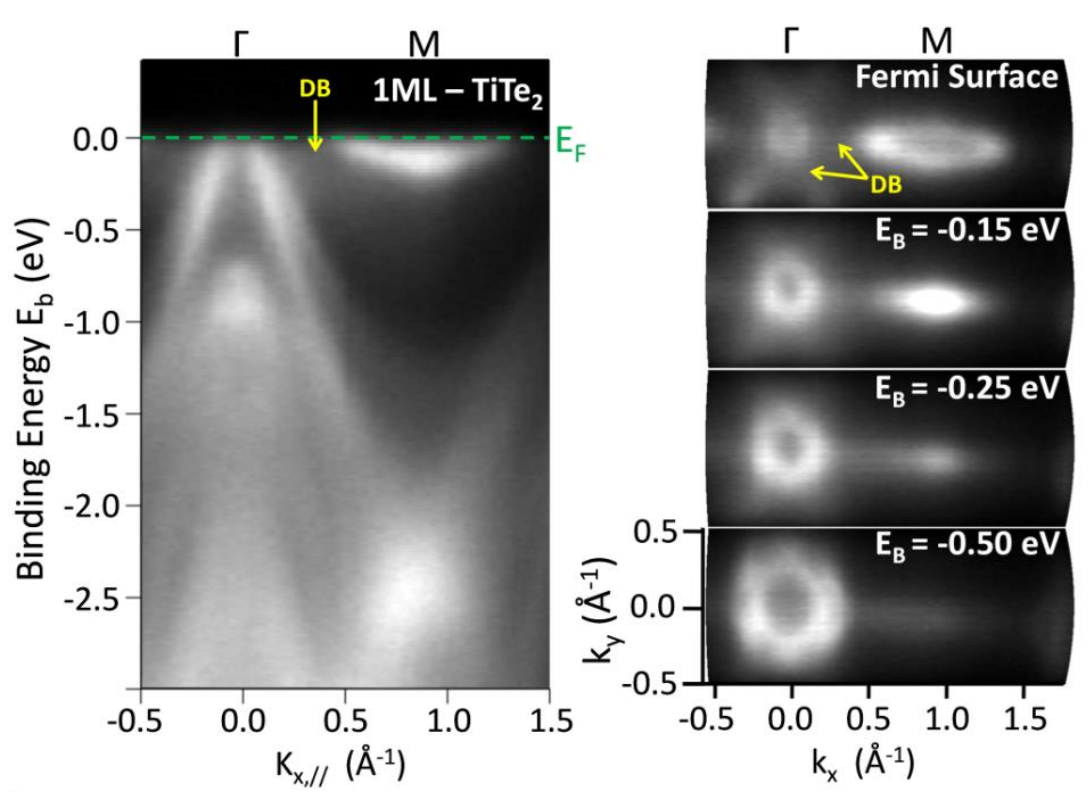

(b)
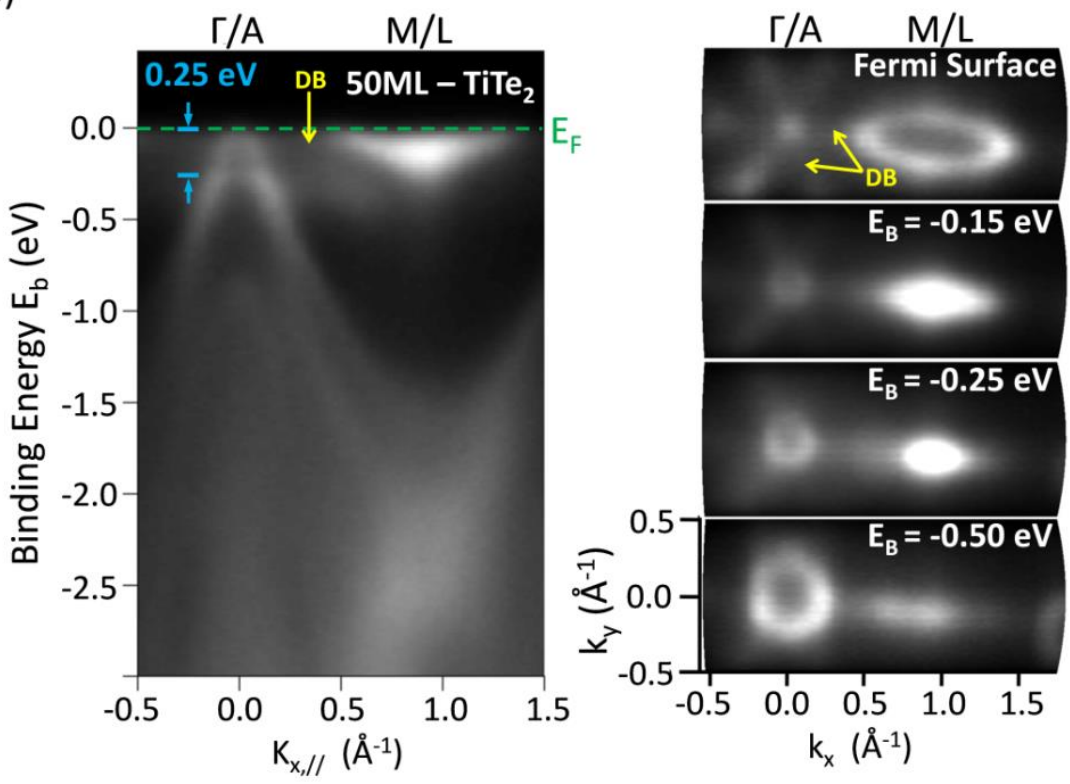

Fig 5: Electronic band structure imaging of CDW TiTe2 by by in-situ ARPES. Energy dispersion $E-k$ along high symmetry directions in the BZ (left panels) and constant energy contour plots $k_{x}-k_{y}$ (right panels) of 1 ML TiTe 2 CDW (a) and 50 ML CDW (b), imaged by in-situ ARPES using He I excitation. Weak and diffuse intensity (blur) connects the $p$-Te hole pocket at $\Gamma$ with the $d$-Ti electron pocket (ellipsoidal contour) at $\mathrm{M}$, forming a dispersionless band (DB) near $\mathrm{E}_{\mathrm{F}}$ which appears as a "star"-like feature in the constant energy contours. 


\section{SUPPLEMENTARY INFORMATION}

\section{In-situ reflection high energy electron diffraction}

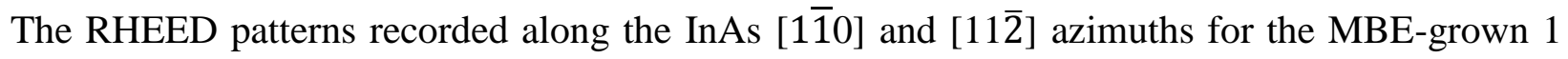
ML and $50 \mathrm{ML} \mathrm{TiTe}_{2}$ as well as for the bare InAs substrate are presented in Figure S1. After insitu substrate preparation, a clean, oxygen and carbon-free In-terminated InAs(111) surface was obtained, as evidenced from the recorded $2 \times 2$ reconstruction, which is attributed to In vacancies $^{1}$. The $2 \times 2$ reconstruction is an indication that the InAs surface is clean and ready for the $\mathrm{TiTe}_{2}$ growth. The streaky RHEED patterns of both $1 \mathrm{ML}$ and $50 \mathrm{ML} \mathrm{TiTe}_{2}$ films indicate very good surface atomic ordering of the epilayers and rotational commensurability. Additional satellite peaks are observed in the vicinity of the main $(1 \times 1)$ streaks of the $1 \mathrm{ML} \mathrm{TiTe} \mathrm{Tetter}_{2}$ visible in off-angle patterns (not shown here), which originate from Moiré interference fringes as described in more detail in the main text in the section discussing STM data (Fig. 2a and b). In

the $1 \mathrm{ML} \mathrm{TiTe}_{2}$ RHEED spectrum, a very faint and broad $\times 2$ reconstruction is also observed mainly in the $[11 \overline{2}]_{\text {InAs }}$ azimuth which is associated to the similarly weak and diffuse STM/ FFT pattern (Fig. 2b).

The same $\times 2$ pattern is clearly visible in both azimuths in the thick (50 ML) film which correlates nicely with the more intense and sharp $2 \times 2$ superstructure in the STM/FFT of the 50 ML TiTe 2 in Fig. $2 d$ attributed to the $\mathrm{TiTe}_{2} \mathrm{CDW}$ structure. These data indicate a much stronger CDW formation in multilayer $\mathrm{TiTe}_{2}$ compared to $1 \mathrm{ML}$ films.

In the same figure $\mathrm{S} 1$, the diffraction pattern of the $50 \mathrm{ML}$ TiTe2 is also shown at elevated temperatures of $400{ }^{\circ} \mathrm{C}$ and $450{ }^{\circ} \mathrm{C}$. At $400 \mathrm{C}$, the $\times 2 \mathrm{CDW}$ streaks are faint but clearly visible On the other hand, at $450{ }^{\circ} \mathrm{C}$, the $\times 2$ superstructure is barely visible (traces can be 
observed only in the $[11 \overline{2}]$ azimuth). This indicates that the transition temperature for the CDW state could be around $400{ }^{\circ} \mathrm{C}$, one of the largest observed for CDWs in 2D TMD materials.

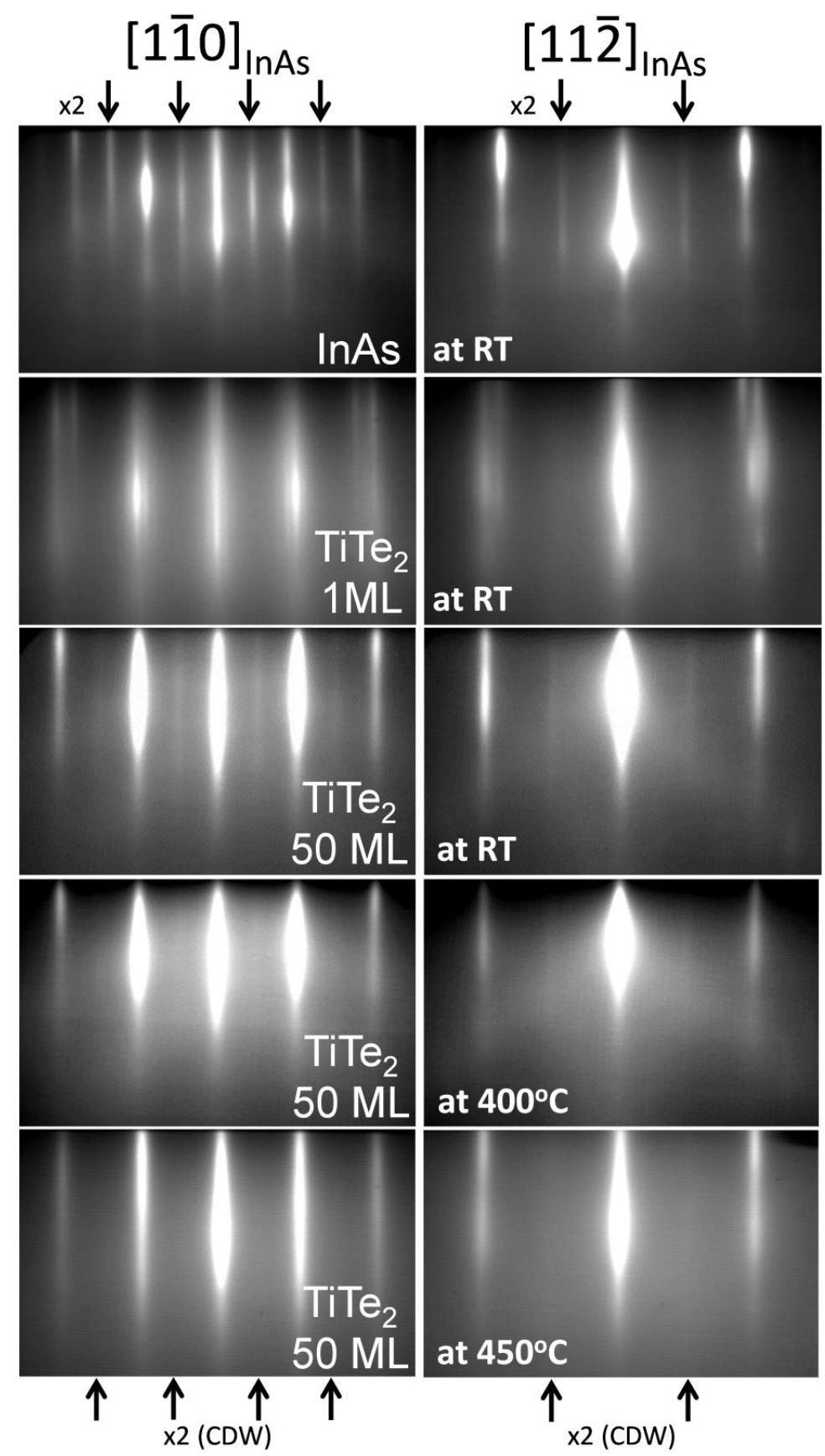

Fig S1. RHEED diffraction patterns obtained at RT of the bare InAs, TiTe ${ }_{2} 1 \mathrm{ML}$ and 50ML films along the $[1 \overline{1} 0]$ and $[11 \overline{2}]$ azimuths of the InAs substrate. For $50 \mathrm{ML} \mathrm{TiTe}_{2}$, the patterns obtained at $400{ }^{\circ} \mathrm{C}$ and $450{ }^{\circ} \mathrm{C}$ are also shown. 


\section{Phonon dispersion using PBE functionals}

The phonon dispersion ${ }^{2}$ is also calculated using PBE functionals ${ }^{3}$ to determine the stability of the $(1 \times 1)$ 1T $\mathrm{TiTe}_{2}$ monolayer (Fig. S2) assuming enlarged lattice parameter $a$ as determined by XRD (see main text). In contrast to the phonon calculations using HSE hybrid functionals, no imaginary frequencies are predicted, indicating that the structure should remain stable since only a small phonon softening at $\mathrm{M}$ occurs. These results are in line with previously published data $^{4}$ for the $1 \mathrm{ML} \mathrm{TiTe}_{2}$ with relaxed lattice parameter $a$, using PBE functionals. This indicates that taking into account electron-correlation effects through HSE functionals is essential for the prediction of a Kohn anomaly at M leading to structural instabilities.

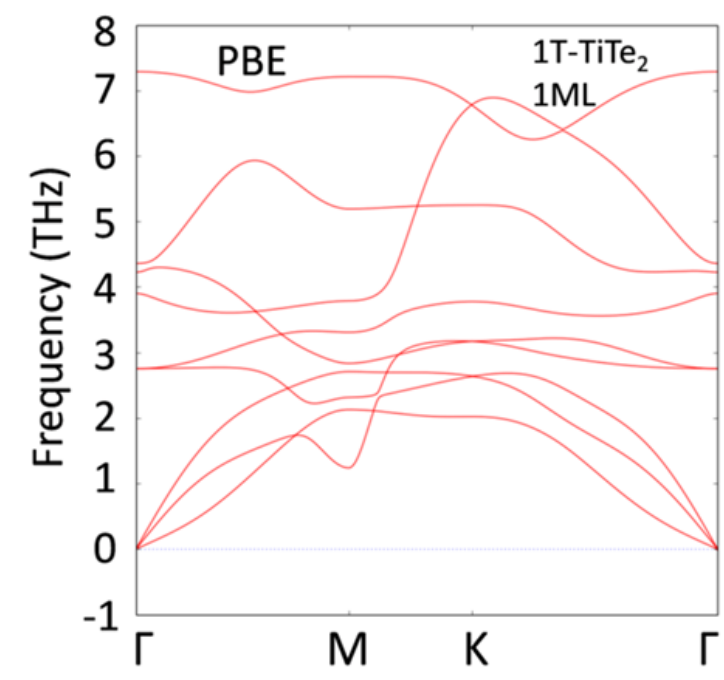

Fig. S2. Phonon frequency dispersion of 1ML 1T TiTe2 along high symmetry points in the first Brillouin zone. 


\section{CDW structural parameters}

\begin{tabular}{|c|c|c|}
\hline \multicolumn{3}{|c|}{$(2 \times 2) \mathrm{CDW}-\mathrm{TiTe}_{2} 1 \mathrm{ML}$ Relaxed } \\
\hline $\mathbf{x}$ & $\mathbf{y}$ & $\mathbf{Z}$ \\
\hline $\mathbf{a}=7.4642171860$ & 0.0000000000 & 0.0000000000 \\
\hline $\mathbf{b}=-3.7321085930$ & 6.4642017024 & 0.0000000000 \\
\hline $\mathbf{c}=0.0000000000$ & 0.0000000000 & 15.0000000000 \\
\hline \multicolumn{3}{|c|}{ Fractional Coordinates } \\
\hline $\begin{array}{ll}\text { Ti1 } & 0.495256275\end{array}$ & 0.252030581 & 0.248314664 \\
\hline $\begin{array}{ll}\mathbf{T i}_{2} & 0.495255589\end{array}$ & 0.763510406 & 0.248314664 \\
\hline $\begin{array}{ll}\text { Ti3 } & 0.983775258\end{array}$ & 0.740549684 & 0.248314664 \\
\hline $\begin{array}{ll}\text { Ti4 } & 0.006736040\end{array}$ & 0.252030045 & 0.248314664 \\
\hline $\begin{array}{ll}\text { Te1 } & 0.162275746\end{array}$ & 0.088754989 & 0.134565175 \\
\hline $\begin{array}{ll}\text { Te2 } & 0.658530712\end{array}$ & 0.082325220 & 0.134565175 \\
\hline Te3 0.664960444 & 0.585009933 & 0.134565175 \\
\hline Te4 0.161922336 & 0.585363388 & 0.135312513 \\
\hline Tes 0.325550795 & 0.415305138 & 0.362064109 \\
\hline Te6 0.331980586 & 0.919050157 & 0.362064109 \\
\hline $\mathbf{T e}_{7} \quad 0.828235567$ & 0.421734899 & 0.362064109 \\
\hline Tes 0.828588963 & 0.918696702 & 0.361316786 \\
\hline
\end{tabular}

Table S1. Atomic positions of $(2 \times 2)$ CDW-TiTe 2 1ML unit cell expressed in fractions of the lattice parameters for the free-standing lattice calculated by DFT 


\begin{tabular}{|c|c|c|}
\hline \multicolumn{3}{|c|}{$(2 \times 2) \mathrm{CDW}-\mathrm{TiTe}_{2} 1 \mathrm{ML}$ Strained } \\
\hline $\mathbf{x}$ & $\mathbf{y}$ & $\mathbf{z}$ \\
\hline $\mathbf{a}=7.6836000000$ & 0.0000000000 & 0.0000000000 \\
\hline $\mathbf{b}=-3.8418000000$ & 6.6541927482 & 0.0000000000 \\
\hline $\mathbf{c}=0.0000000000$ & 0.0000000000 & 15.0000000000 \\
\hline \multicolumn{3}{|c|}{ Fractional Coordinates } \\
\hline $\begin{array}{ll}\text { Ti }_{1} & 0.495256275\end{array}$ & 0.252030581 & 0.248314664 \\
\hline $\begin{array}{ll}\mathbf{T i}_{2} & 0.495255947\end{array}$ & 0.762397528 & 0.248314664 \\
\hline $\begin{array}{ll}\mathbf{T i}_{3} & 0.984888852\end{array}$ & 0.741663218 & 0.248314664 \\
\hline $\begin{array}{ll}\text { Ti4 } & 0.005623817\end{array}$ & 0.252030581 & 0.248314664 \\
\hline $\begin{array}{ll}\text { Te1 } & 0.162326694\end{array}$ & 0.088183500 & 0.137792006 \\
\hline $\begin{array}{ll}\text { Te2 } & 0.659101605\end{array}$ & 0.082946204 & 0.137792006 \\
\hline $\begin{array}{ll}\text { Teз } & 0.664339066\end{array}$ & 0.584959030 & 0.137792006 \\
\hline $\begin{array}{ll}\text { Te4 } & 0.161922574\end{array}$ & 0.585363269 & 0.138474673 \\
\hline $\begin{array}{ll}\text { Te5 } & 0.828185201\end{array}$ & 0.421113431 & 0.358836651 \\
\hline $\begin{array}{ll}\text { Te6 } & 0.326172948\end{array}$ & 0.415876120 & 0.358836651 \\
\hline $\begin{array}{ll}\text { Te}_{7} & 0.331409693\end{array}$ & 0.919100225 & 0.358836651 \\
\hline $\begin{array}{ll}\text { Te8 } & 0.828589797\end{array}$ & 0.918697476 & 0.358153984 \\
\hline
\end{tabular}

Table S2. DFT calculated atomic positions of $(2 \times 2) \mathrm{CDW}_{-} \mathrm{TiTe}_{2} 1 \mathrm{ML}$ unit cell expressed in fractions of the experimental lattice parameters. The latter are deduced from synchrotron GIXD in the present work and are found to be enlarged compared to free-standing values determined from DFT, due to the influence of the substrate. 


\section{STEM Characterization}

An STEM image of the $\mathrm{TiTe}_{2}$ film is shown by Fig. S3a. The epitaxial relationship indicated by the RHEED and GIXD is similarly demonstrated by STEM, and shown by alignment of the $\mathrm{TiTe}_{2}[2 \overline{1} \overline{10}]$ with InAs [110]. The film is $\sim 50 \mathrm{ML}$ of $\mathrm{TiTe}_{2}$, but there are some variations in the thickness as a result of substrate roughness. The InAs substrate has atomic steps across the surface, which disturbs the uniformity of the $\mathrm{TiTe}_{2}$ film as shown by Fig. S3b introducing also some defects. Nevertheless, the interface is abrupt, showing no reaction between the $\mathrm{TiTe}_{2}$ epilayer and the InAs substrate.

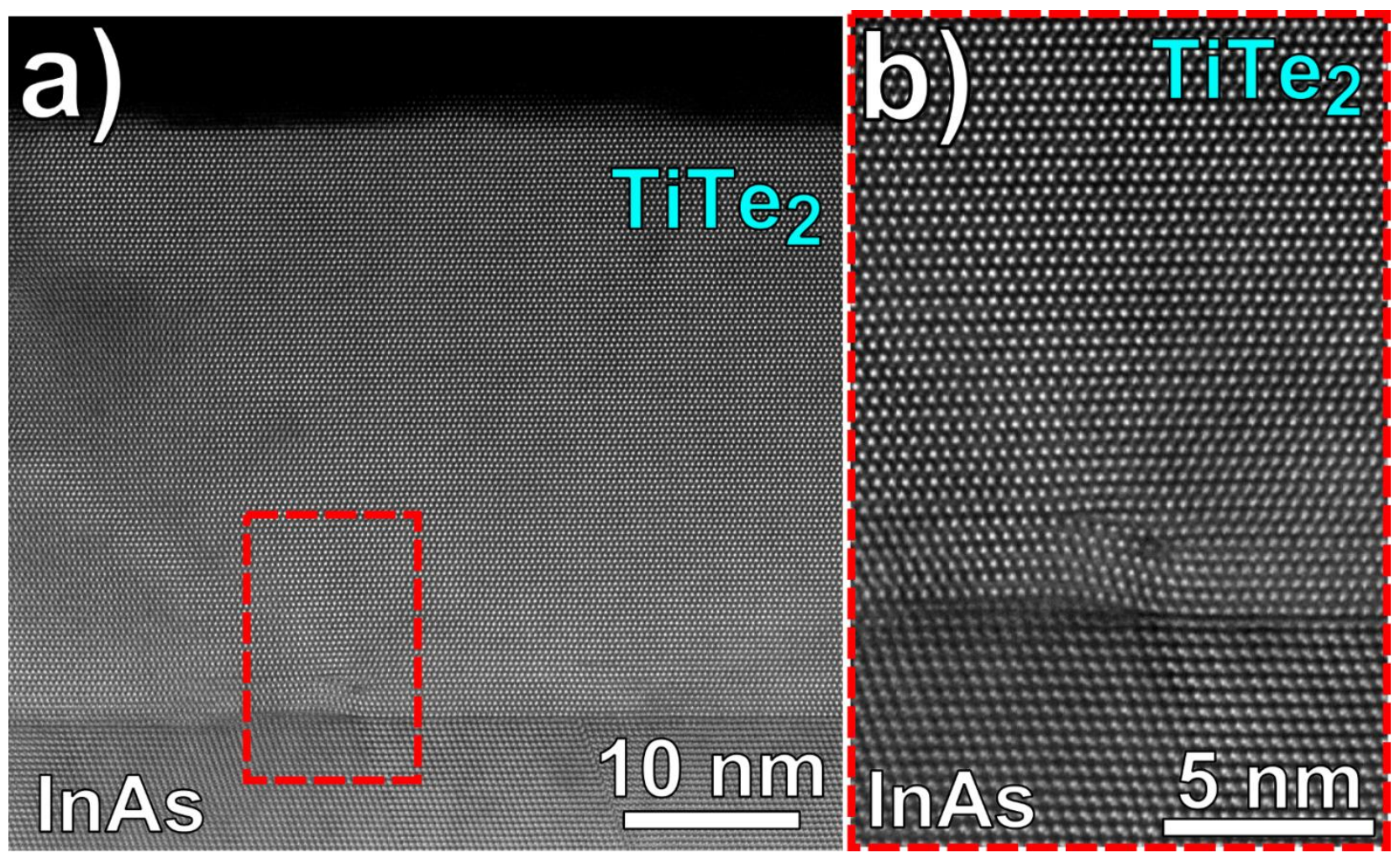

Fig. S3: STEM measurements. (a) STEM image of $\mathrm{TiTe}_{2}$ film on InAs (111) substrate. (b)

Magnified STEM image of $\mathrm{TiTe}_{2}$ film interface with an atomic step present in InAs substrate. 


\section{RSM for $l=0$ and higher diffraction orders of multilayer TiTe $\mathrm{CDW}_{2}$ structure}

The RSM for $l=0.5$ and $l=1$ have already been described in detail in Fig. $4 \mathrm{a}, \mathrm{b}$ in the main text.

Here we complementary give the $l=0$ (a), and higher order diffractions $l=1.5$ (b), $l=2$ (c) and $l=2.5(\mathrm{~d})$.

(a)
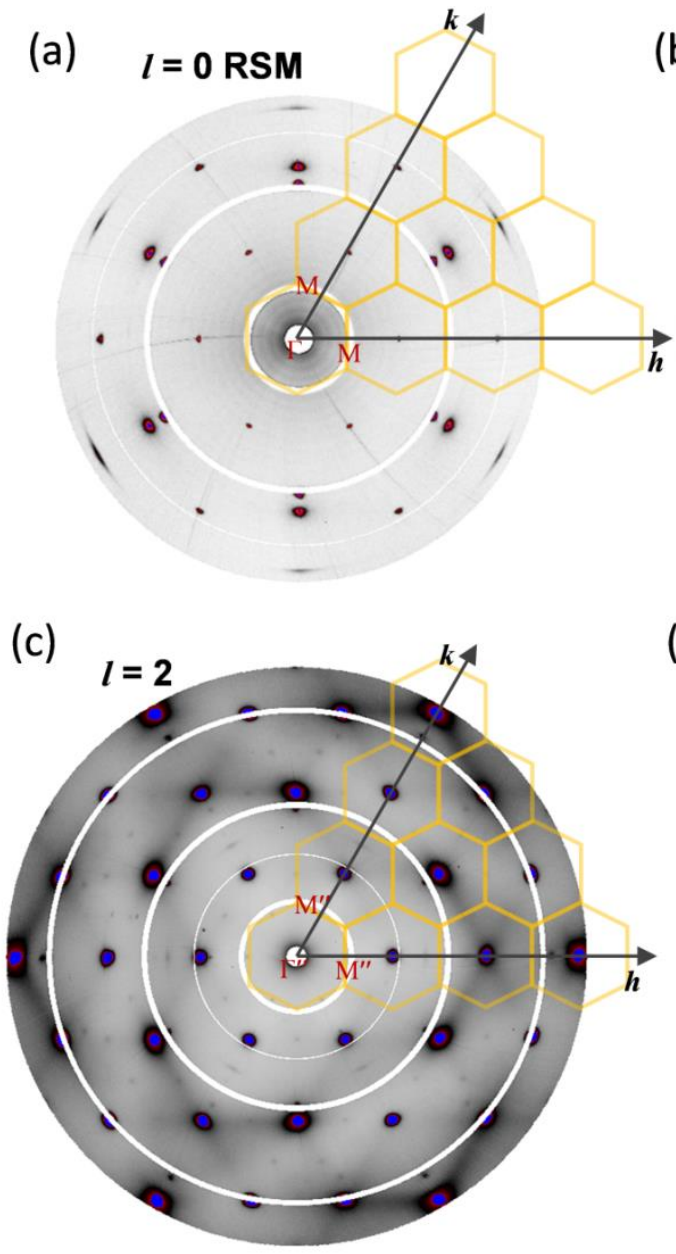

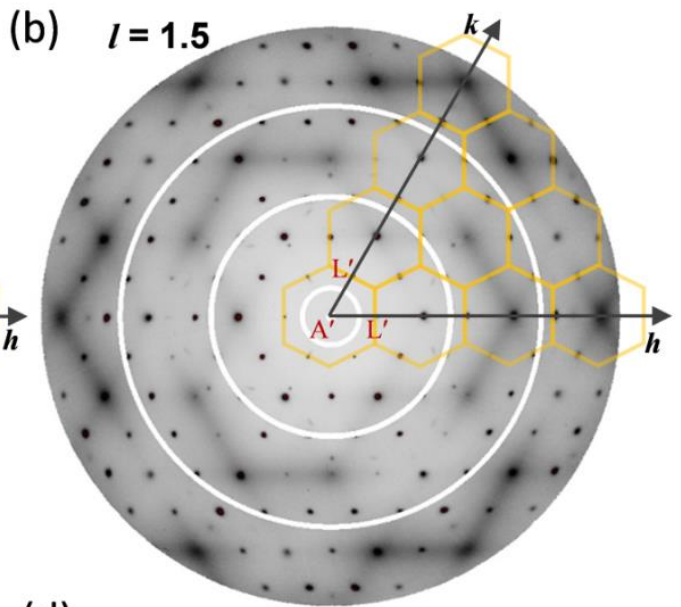

(d)

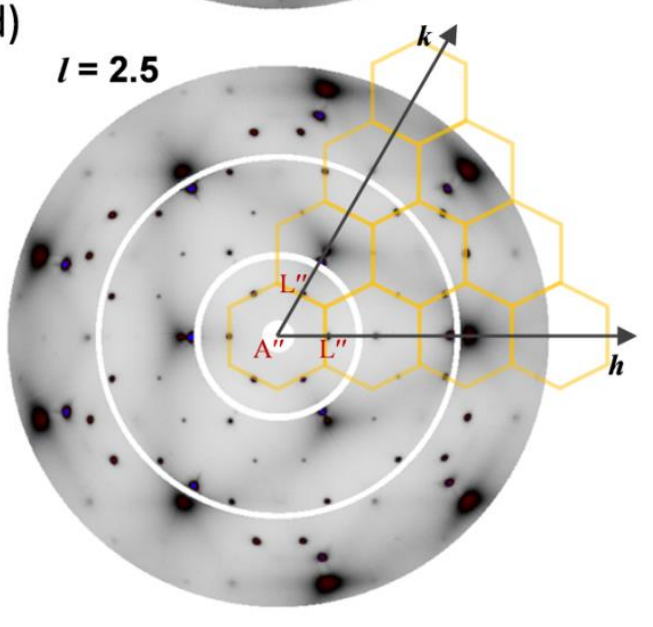

Fig. S4. Reciprocal Space Mapping of 50 ML epitaxial CDW TiTe2 at $l=0$ and higher diffraction orders. The yellow honeycomb-like grid marks the Brillouin zone structure. The letters denote points of high symmetry in the first BZ (unprimed) and in neighboring BZs (primed and double-primed). 
The $l=0$ and $l=2$ RSMs, similar to the $l=1$ RSM (Fig. 4b) are dominated by the $1 \mathrm{~T} \mathrm{TiTe}_{2}$ Bragg peaks at the $\Gamma$ points of the BZ having all indices $h k l$ integer. In the $l=2$ RSM very weak superstructure spots at M points (marked by yellow arrows) are seen similar to the $l=1 \mathrm{RSM}$ in Fig.4b, however their appearance is not confirmed for $l=0$ since the scattered beam is below the sample horizon and thus attenuated by few orders of magnitude. The half integer $l$ RSMs (1.5 and 2.5) are dominated by the CDW superstructure spots, located at the L points of the BZ and having at least one in-plane index $(k$ or $h)$ half integer, similar to $l=0.5 \mathrm{RSM}$ in Fig. 4a (main text).

\section{Simulations of Crystal Trancation Rods (l-scans)}

The structure factor amplitude along crystal truncation rods (CTRs) is calculated as a function of the reciprocal space coordinate $l$ for $(h, k)$ integer (left) and $(h, k)$ half-integer (right) out of plane directions for the model described in, with Debye-Waller factors set equal to zero. When both $h$ and $k$ are integer, only Bragg peaks at integer $l$ coordinates are visible, whereas superstructure peaks are not observed in between. For $(h, k)$ half-integer coordinates instead, peaks occur at half-integer values of $l$ as consequence of the ABA stacking of layers) with intensity of at least one order of magnitude lower than Bragg peaks in the left figure. Moreover, along the same reconstructed directions, additional weaker peaks appear also at integer $l$, in agreement with experimental observations at $\mathrm{M}$ points (see main text). The calculations have been performed with PyRod, software developed at BM32

(ESRF) and based on the well known ROD software ${ }^{4,5}$ for surface diffraction data analysis and processing. 


\section{Diffraction from bulk 1T TiTe2 crystals}

200-300 $\mu$ m-thick bulk TiTe 2 crystals fabricated by chemical vapor transport by HQ Graphene are investigated by GIXD (Fig. S6) to compare with our epitaxial films. All integer $l$ RSMs show the $1 \mathrm{~T} \mathrm{TiTe}_{2}$ bragg peaks at the center ( $\Gamma$ point) of the $\mathrm{BZ}$ as expected. In contrast to the epitaxial films (Figs 4 and S4), the superstructure spots expected at the L points of the BZ are absent from all half-integrer $l$ RSMs (Fig S6) in the bulk $\mathrm{TiTe}_{2}$. This means that bulk $\mathrm{TiTe}_{2}$ is stable in the $1 \mathrm{~T}$ structure and that the $\mathrm{CDW}$ is characteristic of epitaxial films due to the influence from the substrate.
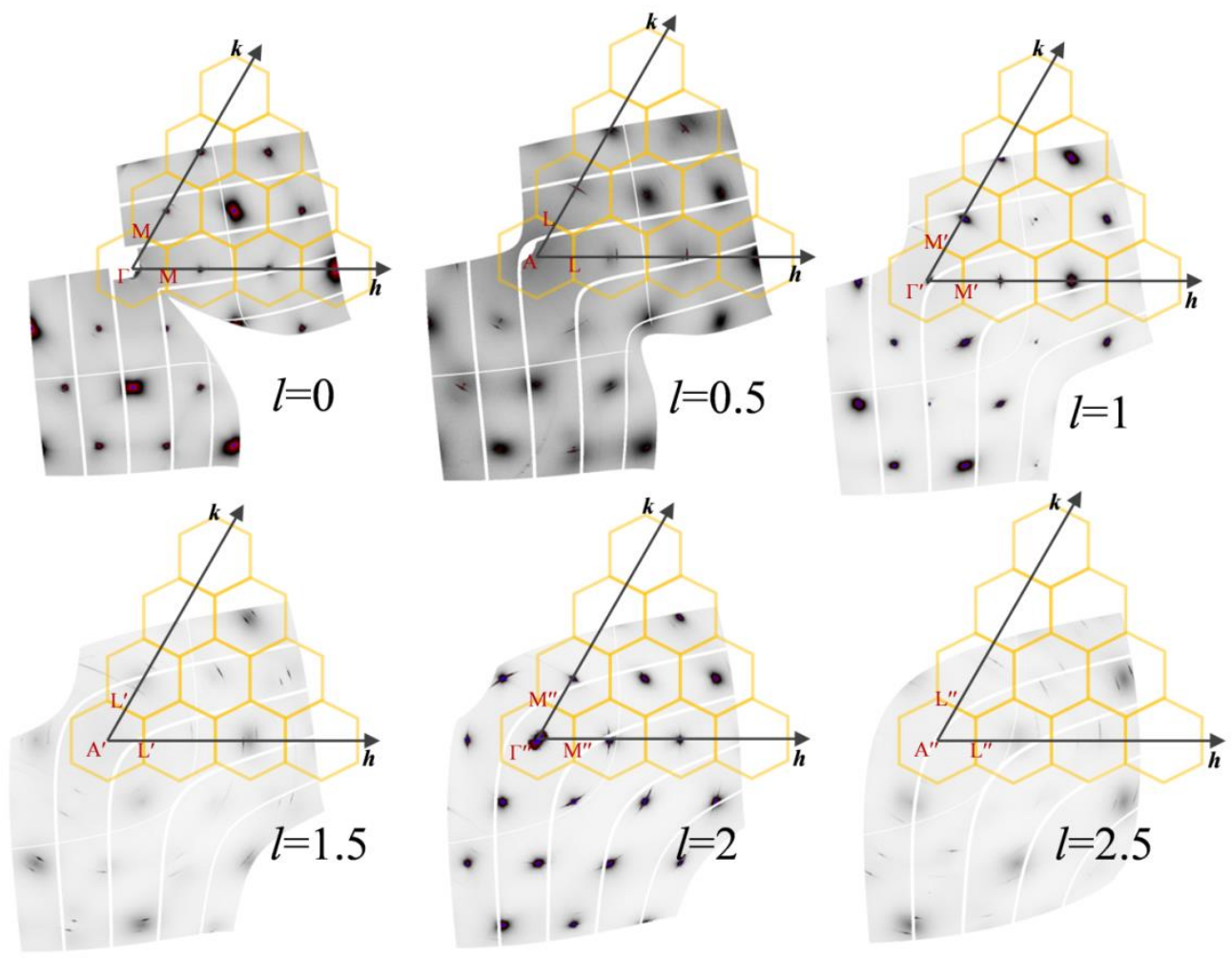

Fig. S5. Reciprocal Space Mapping of bulk 1T TiTe2 for different $l$ values. The yellow honeycomb-like grid marks the brillouin zone structure. The letters denote points of high 
symmetry in the first BZ (unprimed) and in neighboring BZs (primed and double-primed).

\section{GIXD-Rocking curves and mosaicity}

Furthermore, low in-plane mosaic spread of $1.09^{\circ}$ and $1.59^{\circ} \mathrm{FWHM}$ for $50 \mathrm{ML}$ and $1 \mathrm{ML} \mathrm{TiTe}_{2}$, respectively are deduced from the rocking curves of the $\mathrm{TiTe}_{2}$ (300) diffraction (Bragg) peaks.

(a)

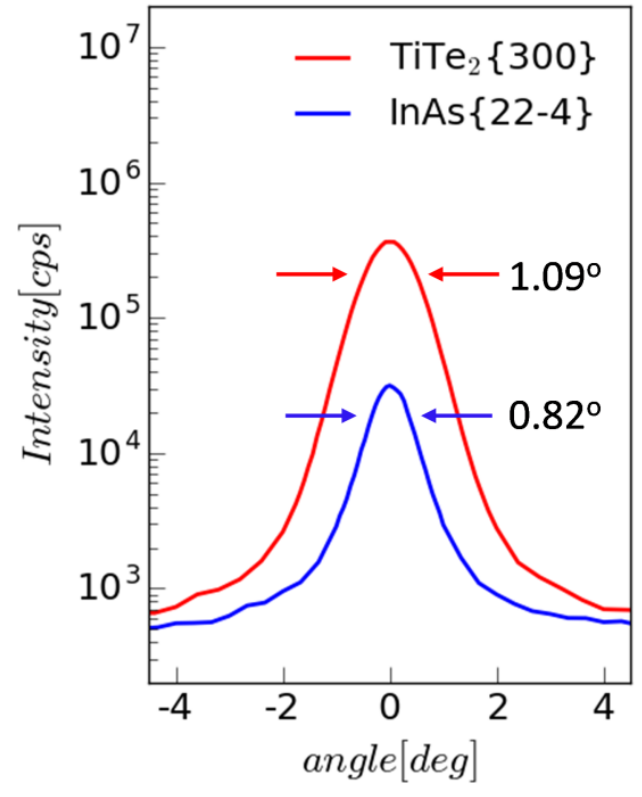

(b)

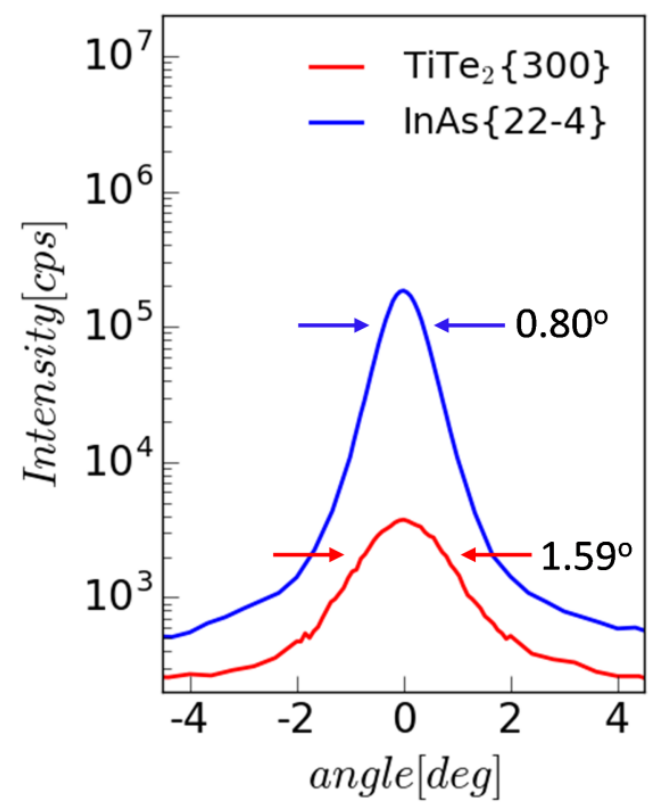

S6. Rocking curves. The data are shown in logarithmic scale around the $\operatorname{TiTe}_{2}(300)$ and the InAs (22-4) Bragg peaks for $50 \mathrm{ML}$ (a) and $1 \mathrm{ML}$ (b) $\mathrm{TiTe}_{2}$ films. The arrows show where the FWHM is measured.

The latter values are better than those obtained from the InAs/ZrTe $(1 \mathrm{ML})^{7}$ and $\mathrm{InAs} / \mathrm{MoTe}_{2}{ }^{8}$ systems, and comparable to the $0.80^{\circ}-0.82^{\circ}$ mosaic spread of the InAs substrate (Fig. S3), indicating $\mathrm{TiTe}_{2}$ films of high epitaxial quality which is essentially limited by the heteroepitaxial InAs template quality 


\section{Electronic band structure of CDW TiTe2: The dispersionless band}

As already pointed out in Figure 5, ARPES shows a dispersionless band (DB) with a diffuse intensity distributed along $\Gamma(\mathrm{A})-\mathrm{M}(\mathrm{L})$ of the BZ. This band indicates localized electrons likely due to strong-electron phonon interaction that forms a polaron state ${ }^{9}$. This polaron formation is supportive of the assumption that the CDW is driven by soft phonons with wavevectors $\vec{q}=\overrightarrow{\Gamma L}$ and $\vec{q}=\overrightarrow{\Gamma M}$ (See discussion and conclusions section), therefore it deserves more attention. Below in Fig S7, the linescans at $\mathrm{k}_{\mathrm{y}}=0$ are presented to better illustrate the dispersionless band (shown by the red arrow) which connects the $\Gamma(\mathrm{A})$ and $\mathrm{M}(\mathrm{L})$ points The intensity peaks at $\mathrm{E}_{\mathrm{F}}$ but it also has finite intensity for slightly smaller energies about $0.2 \mathrm{eV}$ below $\mathrm{E}_{\mathrm{F}}$.

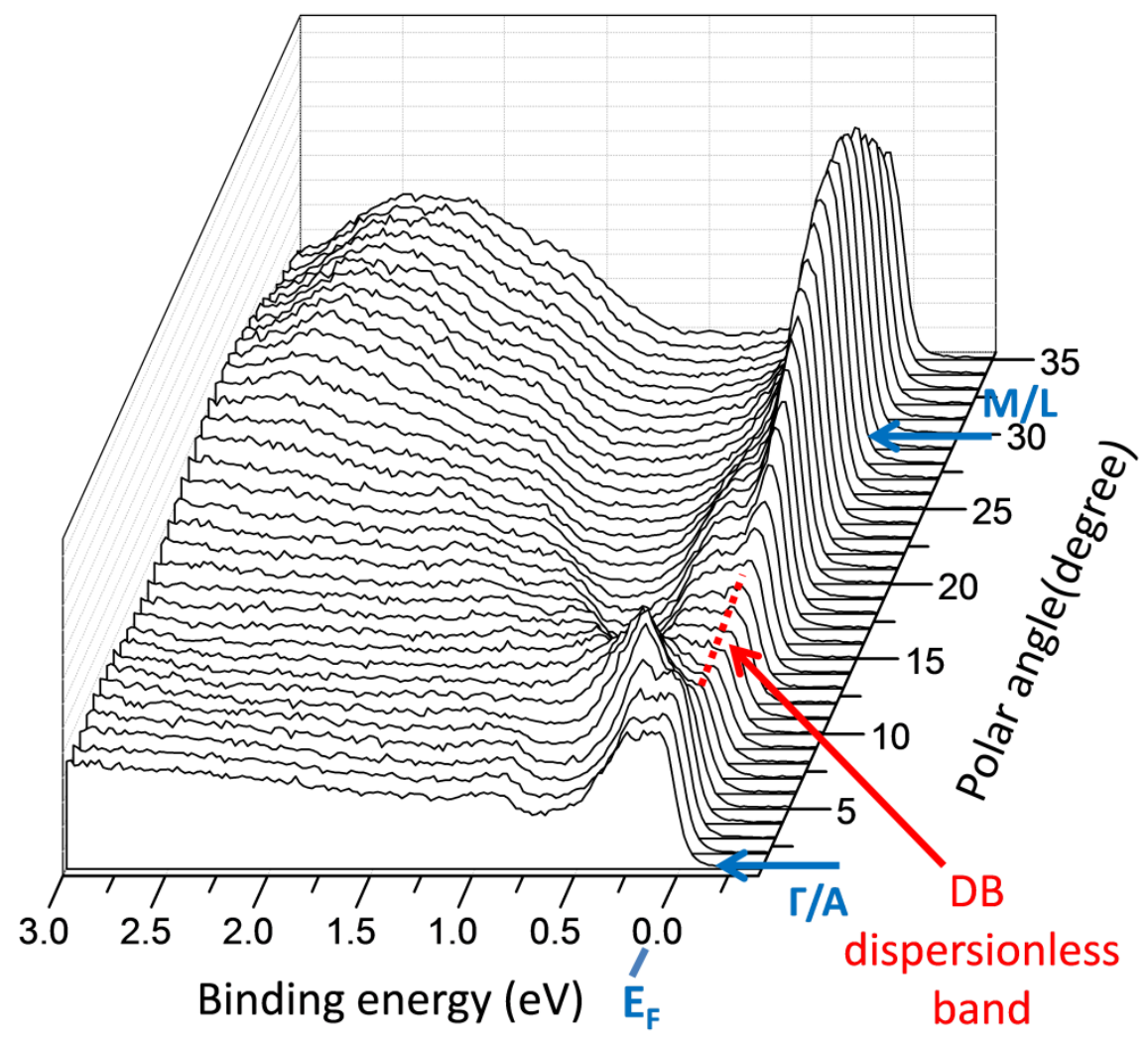

S7. ARPES line scans of CDW multilayer TiTe2. The spectra $E-k_{x, / /}$ at $k_{y}=0$ show the 
dispersionless band (DB) marked by red arrow and red dashed line

\section{References (Supplementary)}

1. Tagushi, A., Kanisawa, K. Stable reconstruction and adsorbates of InAs(111)A surface Appl. Surf. Sci. 252, 5263-5266 (2016).

2. Togo, A. \& Tanaka, I. First principles phonon calculations in materials science. Scr. Mater. 108, 1-5 (2015).

3. Perdew, J. P., Burke, K. \& Ernzerhof, M. Generalized gradient approximation made simple. Phys. Rev. Lett. 77, 3865-3868 (1996).

4. Chen, P., Pai, W. W., Chan, Y.-H., Takayama, A., Xu, C.-Z., Karn, A., Hasegawa, S., Chou, M. Y., Mo, Z., Fedorov, A.-V. \& Chiang, T.-C. Emergence of charge density waves and a pseudogap in single-layer TiTe 2 . Nat. Commun. 8, 516 (2017).

5. Drnec, J., Zhou, T., Pintea, S., Onderwaater, W., Vlieg, E., Renaud, G. \& Felici, R. Integration techniques for surface X-ray diffraction data obtained with a two-dimensional detector. J. Appl. Cryst. 47, 365-377 (2014).

6. Vlieg, E. ROD: a program for surface X-ray crystallography. J. Appl. Cryst. 33, 401-405 (2000).

7. Tsipas, P., Tsoutsou, D., Fragkos, S., Sant, R., Alvarez, C., Okuno, H., Renaud, G., Alcotte, R., Baron, T. \& Dimoulas, A. Massless Dirac Fermions in $\mathrm{ZrTe}_{2} \mathrm{Semimetal}$ Grown on InAs(111) by van der Waals Epitaxy. ACS Nano 12, 1696-1703 (2018).

8. Tsipas, P., Fragkos, S., Tsoutsou, D., Alvarez, C., Sant, R., Renaud G., Okuno, H., Dimoulas, A. Direct observation at room temperature of the orthorhombic Weyl semimetal phase in thin epitaxial MoTe 2. Adv. Funct. Mater. 28, 1802084 (2018). 
9. De Boer, D. K. G., van Bruggen, C. F., Bus, G. W., Coehoorn, R., Haas, C., Sawatzky, G. A., Myron, H. W., Norman, D. \& Padmore H., Titanium ditelluride: Band structure, photoemission, and electrical and magnetic properties, Phys. Rev B 29, 6797 (1984). 